\title{
Extremely hard GRB spectra prune down the forest of emission models
}

\author{
G. Ghirlanda ${ }^{1}$, A. Celotti ${ }^{1}$, and G. Ghisellini ${ }^{2}$ \\ 1 SISSA/ISAS, via Beirut 2-4, 34014 Trieste, Italy \\ 2 Osservatorio Astronomico di Brera, via Bianchi 46, 23807 Merate, Italy
}

Received 4 November 2002 / Accepted 20 May 2003

\begin{abstract}
We consider the evidence for very hard low energy spectra during the prompt phase of Gamma-Ray Bursts (GRB). In particular we examine the spectral evolution of GRB 980306 together with the detailed analysis of some other bursts already presented in the literature (GRB 911118, GRB 910807, GRB 910927 and GRB 970111), and check for the significance of their hardness (i.e. extremely steep spectral slopes below the $E F_{E}$ peak) by applying different tests. These bursts, detected by the Burst And Transient Source Experiment (BATSE) in the $\sim 30 \mathrm{keV}-2 \mathrm{MeV}$ energy range, are sufficiently bright to allow time resolved spectral studies on time intervals of the order of tenths of a second. We discuss the hard spectra of these bursts and their evolution in the context of several non-thermal emission models, which all appear inadequate to account for these cases. The extremely hard spectra, which are detected in the early part of the BATSE light curve, are also compared with a black body spectral model: the resulting fits are remarkably good, except for an excess at high energies (in several cases) which could be simply accounted for by the presence of a supra-thermal component. The findings on the possible thermal character of the evolving spectrum and the implications on the GRB physical scenario are considered in the frameworks of photospheric models for a fireball which is becoming optically thin, and of Compton drag models, in which the fireball boosts "ambient" seed photons by its own bulk motion. Both models, according to simple estimates, appear to be qualitatively and quantitatively consistent with the found spectral characteristics, although their possible caveats are discussed.
\end{abstract}

Key words. gamma rays: bursts - methods: data analysis - radiation mechanisms: non-thermal

\section{Introduction}

Since the discovery of these mysterious and puzzling cosmic explosions, the time resolved spectral analysis of Gamma-Ray Bursts (GRBs) has been a testing ground-floor for the models proposed for the $\gamma$-ray prompt emission (Ford et al. 1995; Crider et al. 1997a). In general GRBs have a continuous curvature spectrum which is usually characterized (Band et al. 1993) by two smoothly joining components defining a peak (in a $E F_{E}$ representation of the power per unit logarithmic energy interval) at energies around $200 \mathrm{keV}$. The low energy asymptotic spectrum can be modeled as a power law function in the photon distribution $N(E) \propto E^{\alpha}$ (i.e. $E F_{E} \propto E^{\alpha+2}$ ), where $E$ indicates the photon energy. The high energy spectral component can be described in most cases by a steep power law $E^{\beta}$ (with $\beta<\alpha$ ) or an exponential cutoff. The typical values of $\alpha$ and $\beta$ are widely distributed around -1 and -2.5 respectively (Preece et al. 2000; see also Fig. 1).

In particular, different authors (Crider et al. 1999; Preece et al. 1998b) have stressed the importance of studying the low energy component of GRB spectra which is one of the better determined and accessible observables. Crider et al. (1997b) showed that the low energy spectrum evolves in time and that

Send offprint requests to: G. Ghirlanda, e-mail: ghirland@sissa. it the distribution of the spectral index $\alpha$ extends above -1 and in some cases can be $\alpha \geq 1$.

The most popular radiative process proposed for interpreting GRB spectra is synchrotron emission by relativistic electrons in intense magnetic fields (Rees \& Mészáros 1994; Katz 1994; Tavani 1996). Optically-thin synchrotron theory predicts (Katz 1994) that the low energy photon spectrum can not be harder than $N(E) \propto E^{-2 / 3}$. Nonetheless, different authors (Crider et al. 1997b; Preece et al. 1998b, 2002) have found violations of this limit in many observed spectra. Following these inconsistencies between the standard synchrotron model and observations, alternative scenarios have been proposed, such as jitter radiation (Medvedev 2001); synchrotron emission from particles with an anisotropic pitch angle distribution (Lloyd \& Petrosian 2000, 2002); thin/thick synchrotron emission from a stratified region (Granot et al. 2000); synchrotron self-Compton or inverse Compton off photospheric photons (Mészáros \& Rees 2000); Compton drag (Lazzati et al. 2000; Ghisellini et al. 2000); Comptonization of low energy photons by thermal or quasi-thermal particles (Liang et al. 1997; Ghisellini \& Celotti 1999). The physical parameters of these models can be tuned and combined to justify the principal observed temporal and spectral characteristics of GRBs, producing a considerable number of plausible spectral shapes. 
However, the most extreme (in this case hardest) low energy spectra and their evolution can be used to rule out or constrain some of these possibilities.

Another relevant aspect of GRB spectra is their possible thermal character. In fact, the fireball model (Goodman 1986; Paczynski 1986) naturally predicts thermal radiation when the fireball becomes transparent. The lack of observational evidence of thermal spectra motivated the proposal of the internal shock model (Rees \& Mészáros 1994) in which the fireball energy could be efficiently extracted and transformed into radiation with a non-thermal spectrum as observed in most bursts. Nonetheless, evidence for possible thermal spectra in some GRBs has been reported by Preece (2001) for GRB 970111 and Schaefer et al. (poster P-56 presented at the Rome 1998 Whorkshop on GRBs, private communication). Furthermore Blinnikov et al. (1999) propose that also the spectra observed in most GRBs could be interpreted as superposition of black body spectra with different temperatures. From a theoretical point of view thermal emission from the fireball is expected if the dominant acceleration agent is internal pressure (Daigne \& Mochovitch 2002). Alternatively, magnetic acceleration (e.g. by Poynting flux) would determine a non-thermal spectrum (Drenkhahn \& Spruit 2002; but see also Ruffini et al. 2002). The analysis of spectra with a possible thermal character can therefore have important implications for the understanding of the nature of the fireball acceleration mechanism.

To the aim of testing the validity/generality of the proposed models we have then searched among the $156 \mathrm{GRBs}$ of the published spectral catalog of Preece et al. (2000) the bursts with a low energy time resolved spectrum harder than $N(E) \propto E^{0}$, which represents a limit for most of the above models (see Sect. 2). We found two extremely hard bursts: GRB 911118 (BATSE trigger 1085), whose peak energy evolution has been reported in the spectral catalog by Ford et al. (1995), and a new case, GRB 980306 (trigger 6630). These two GRBs are carefully studied here in terms of their low energy spectral hardness. Their spectral evolution on timescales of few tenths of a second is presented in Sects. 4.1 and 4.2, particularly regarding the slope of the low energy spectral component. We also found GRB 910807 (trigger 647) and GRB 970111 (trigger 5773) whose spectral properties have already been presented in the literature by Crider et al. (1997b, 1997a, 2000) and Frontera et al. (2000) (only for GRB 970111). We included also GRB 910927 (trigger 829) reported by Crider et al. (1997b, 2000) which is not present in the spectral catalog of Preece et al. (2000). These bursts have been re-analyzed in order to test the reliability of their low energy spectral hardness (Sect. 4.4). In fact, we present the tests that we performed in order to determine the statistical robustness of the spectral hardness, and in particular a model independent approach consisting in the comparison of each spectrum with a template one of given hardness (Sect. 4.3). The evidence that, at least at the beginning of the bursts, the spectra are extremely hard suggested their comparison with a black body model. This part of the analysis is presented in Sect. 4.5. The comparison with the low energy spectral limits predicted by different models (briefly recalled in Sect. 2 together with the previous evidence of hard spectra) is the content of the discussion (Sect. 5), where

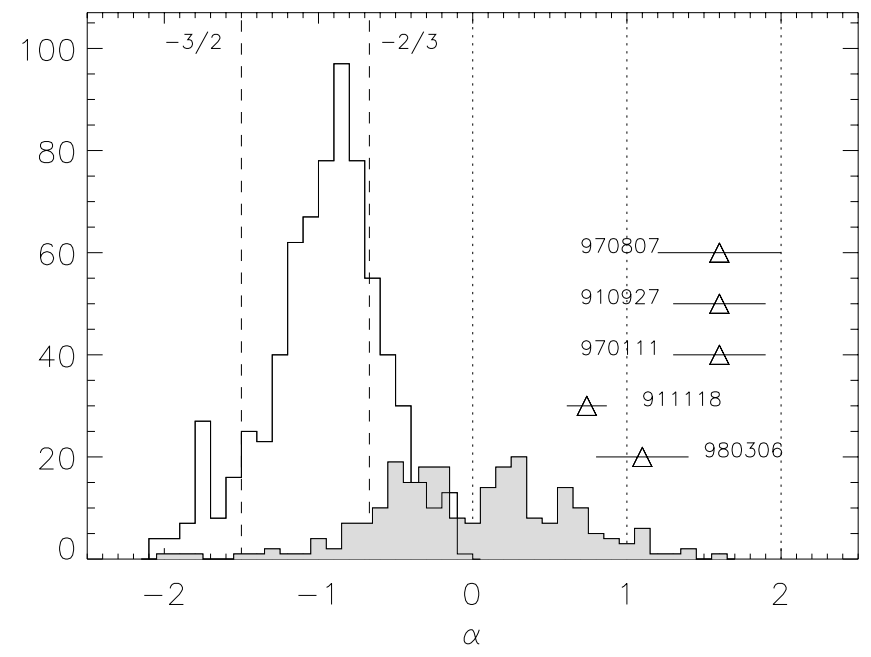

Fig. 1. Low energy spectral index distribution from Ghirlanda et al. (2002). The vertical lines represent the limits of the emission models reported in Table 1 and plotted in Fig. 2. The triangles represent the maximum values found from the spectral analysis of the bursts presented in this work along with their $90 \%$ confidence interval (horizontal bars). The gray histogram shows the distribution of the low energy spectral index found from the time resolved spectral analysis of the bursts presented in the text.

also tentative interpretations of the initial quasi-thermal spectral evolution, in the context of the hot fireball model and of the the Compton drag model, are presented. We draw our conclusions in Sect. 6.

\section{Spectral slopes and emission models}

The distribution of the low energy photon spectral index $\alpha$ obtained from the time resolved spectral analysis of a sample of bright BATSE bursts (Ghirlanda et al. 2002) is reported in Fig. 1. The majority of GRB spectra (Preece et al. 2000; Ghirlanda et al. 2002) have a low energy power law spectral index $-3 / 2 \leq \alpha \leq-2 / 3$, i.e. within the limits predicted by the optically thin synchrotron model (Fig. 2, dashed lines; see also Katz 1994), but there is a non negligible fraction of bursts $(\sim 15 \%$, see also Preece et al. 2000) with a low energy spectrum harder than $N(E) \propto E^{-2 / 3}$. The spectral analysis of a sample of GRBs by Crider et al. (1997b) also revealed that in $60 \%$ of their cases the spectrum evolves with time. Moreover, the spectrum integrated over the pulse rise phase is harder than $E^{-2 / 3}$ in $40 \%$ of their bursts and can be as hard as $N(E) \propto E^{1}$ (i.e. $E F_{E} \propto E^{3}$ ). For comparison the distribution of the $\alpha$ parameter for the hard spectra of the bursts considered in this work is also reported (gray histogram) in Fig. 1. Note that they contribute to extend the distribution toward positive $\alpha$ values up to $\sim 1.5$.

Let us here just recall that this evidence is hardly reconcilable with the simplest formulation of the synchrotron model and some alternatives have been proposed to account for these observations within the frame of this emission process (Papathanassiou 1999; Granot et al. 2000). Lloyd \& Petrosian (2000) propose a scenario in which electrons have a small pitch angle distribution (SPD in Fig. 2), extending the range of 


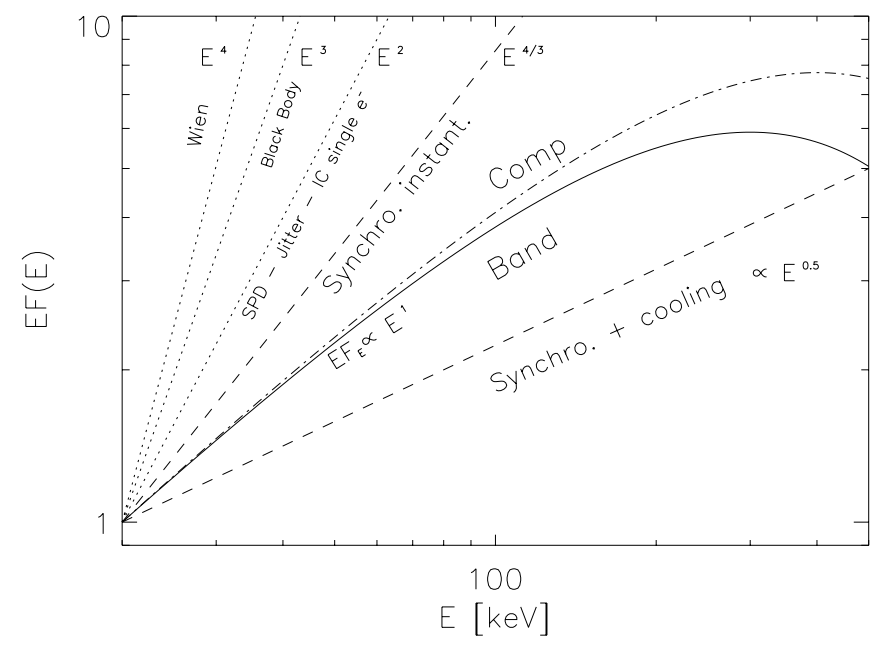

Fig. 2. Low energy spectral limits for different emission models (see Table 1). Examples of the BAND (solid line) and COMP model (dotdashed line) with the low energy photon spectral index fixed at -1 (i.e. $E F_{E} \propto E^{1}$ ) are also represented for comparison.

Table 1. Low energy limiting photon spectral index $\alpha$ [i.e. $\left.N(E) \propto E^{\alpha}\right]$ for various emission models. For clarity the indices for the energy spectrum $F(E)$ and for its $E F_{E}$ representation are also reported.

\begin{tabular}{cccl}
\hline \hline$\alpha$ & $\alpha+1$ & $\alpha+2$ & \\
$N(E)$ & $F(E)$ & $E F_{E}$ & model/spectrum \\
\hline$-3 / 2$ & $-1 / 2$ & $1 / 2$ & Synchrotron emission with cooling \\
-1 & 0 & 1 & Quasi-saturated Comptonization \\
$-2 / 3$ & $1 / 3$ & $4 / 3$ & Instantaneous synchrotron \\
0 & 1 & 2 & $\begin{array}{l}\text { Small pitch angle/jitter } \\
\end{array}$ \\
1 & 2 & 3 & Inverse Compton by single $\mathrm{e}^{-}$ \\
2 & 3 & 4 & Wien Body \\
\hline
\end{tabular}

possible low energy spectral indices produced via synchrotron to the limit $\alpha \sim 0$. The same limiting slope can be obtained in the "jitter" radiation theory, which is based on synchrotron emission in a non uniform magnetic field with inhomogeneities on length scales smaller than the electron gyro-radius (Medvedev 2001). Slopes even harder (i.e. $\alpha \sim 1$ ) may instead correspond to thermal radiation, such as a portion of a black body spectrum (Mészáros \& Rees 2000) or saturated Comptonization spectra with Wien peaks which can be as hard as $\alpha \sim 2$ (Liang et al. 1997; Ghisellini \& Celotti 1999; Ghisellini et al. 2000).

A detailed discussion of these models and their comparison with our observational findings are presented in Sect. 6. As reference for the following sections we schematically summarize the model predictions in Table 1, and display in Fig. 2 their typical low energy limiting slopes in a $E F_{E}$ representation.

\section{Data analysis}

We have analyzed the Large Area Detector (LAD) High Energy Resolution Burst (HERB) data which have a high count rate, due to the LAD large effective area, and are suited for the spectral analysis of GRBs for which a moderate energy resolution is sufficient to study the typical broad band continuum (Preece et al. 1998a).

We selected the data from the most illuminated detector which has the highest signal to noise ratio $(\mathrm{S} / \mathrm{N})$, and maintained the instrumental time binning corresponding (in the best cases) to single time resolved spectra accumulated for $128 \mathrm{~ms}$, to follow the spectral variations on the smallest possible timescale (thanks to the high $\mathrm{S} / \mathrm{N}$ there was no need to rebin the data before fitting). Each spectrum has been fitted from $E_{\min } \sim 28 \mathrm{keV}$ to $E_{\max } \sim 1800 \mathrm{keV}$ which define the typical energy window for the LAD data (Preece et al. 1998a, 2000).

The spectral analysis has been carried out using SOAR (Ford et al. 1993) as a quick look tool for the spectral evolution and then XSPEC (v11.1) for the individual spectral fitting of time resolved spectra. The background, to be subtracted to each time resolved spectrum, has been calculated as the average over a selected number of background spectra accumulated before and after the trigger.

In the data analysis we have used the standard forward folding technique to fit the model spectral functions (convolved with the detector response matrix) to the observed spectra. As explained in Sect. 4, we also used the instrumental spectra in the comparison with a simulated spectrum in order to verify the robustness of our results.

\subsection{Fitting models}

We fitted the most commonly adopted GRB spectral models: the BAND (Band et al. 1993) and COMP model, represented by a smoothly connected double power law or by a single power law ending with an exponential cutoff, and the sharply connected double power law model (BPLW). The choice of these models is motivated by the fact that they characterize the low energy part of the spectrum with a spectral parameter (i.e. the spectral index $\alpha$ ) which can be simply compared with the predictions of the different emission processes. For a detailed description of these spectral functions see Preece et al. (2000).

Due to the extreme hardness of the low energy spectrum of these bursts, we decided also to verify if thermal, black body like, emission was consistent with the low energy data. We considered only the time resolved spectra which have a low energy spectral index $\alpha \geq 0.5$ when fitted with the BAND or COMP models. This indicative value, which is softer than $\alpha=1$ (i.e. the Rayleigh-Jeans limit of a black body spectrum) has been chosen to: a) account for typical errors in the determination of $\alpha$ with the BAND or COMP models; b) include the possibility that the spectrum could be softer than $\alpha=1$, if its peak is at low energies, due to the spectral curvature (see also Sect. 4.5).

The result of each fit was then considered acceptable if the reduced $\chi_{r}^{2}$ was lower than 1.5 for typically $\sim 110$ degrees of freedom (d.o.f.): in fact the statistical probability of having a better fit is around 0.5 if the reduced $\chi_{r}^{2}$ is around 1 , but a limiting value of $\chi_{r}^{2} \sim 1.5$ is suggested if one considers that $\chi_{r}^{2}$ depends on the quality of the data (Bevington \& Robinson 1992). In addition we visually inspected the data-to-model ratio to look for possible systematics (i.e., sequence of points significantly above or below unity). We discarded the black body as a 
good fit when an excess of flux occurred at low energies (i.e. in the $E^{2}$ part of the spectrum), while we allowed for (a moderate) deviation from unity at high energies (i.e. in the exponential part). This latter choice reflects the possible (or even likely) situation of having a "supra-thermal" tail to a Maxwellian distribution in quasi-thermal plasmas, or a multi-temperature black body emission as proposed by Blinnikov et al. (1999).

\section{Results}

Section 4.1 deals with the low energy spectrum of GRB 980306. In particular we focus on the early phase of this burst when the spectrum is extremely hard and report the time evolution of its low energy spectral component. The detailed spectral analysis of GRB 911118 (also reported by Ford et al. 1995 only in terms of the time evolution of the peak energy) is presented in Sect. 4.2 with emphasis (again) on the low energy spectral index evolution. In Sect. 4.3 we describe the tests we performed on the raw data to check for the significance of these results on the spectral hardness. In Sect. 4.4 we briefly discuss other 3 bursts (GRB 910807, GRB 910927 and GRB 970111) with hard spectra at low energies which were already presented in the literature. We have re-analyzed them, applying the tests described in Sect. 4.3, to verify their low energy hardness. In particular for GRB 910807 we have extended the time analysis beyond the first $5 \mathrm{~s}$ that were previously studied by Ryde \& Svensson (1999). The results relative to the black body fits are reported separately in Sect. 4.5.

\subsection{GRB 980306}

GRB 980306 represents a new case of hard burst. It is a single peak burst (Fig. 3, top panel) with duration of $\sim 6 \mathrm{~s}$, peak flux of $(17.2 \pm 0.4)$ phot $\mathrm{cm}^{-2} \mathrm{~s}^{-1}$ at $t_{\text {peak }} \sim 2.2 \mathrm{~s}$ and a total (100300) $\mathrm{keV}$ fluence of $\sim 10^{-5} \mathrm{erg} \mathrm{cm}^{-2}$. ${ }^{1}$ This burst was already present in the Preece et al. (2000) spectral catalogue but, as it had been fitted with a BPLW model only, it resulted softer than what we have found by fitting its spectra with the COMP model. In fact, as already noticed in Ghirlanda et al. (2002), a fit of a model with a sharp break, like the BPLW, to a smoothly curved spectrum, although can still give acceptable fits, tends to underestimate the low energy spectral hardness.

We present, for the first time, its hardness evolution, during the first $6 \mathrm{~s}$, divided in 14 time resolved spectra. In particular, we focus on the extremely hard low energy spectral component. The spectral fits with the different models show that the spectra are better represented by the COMP model, since the high energy spectrum is very steep (i.e. steeper than a power law with spectral index $\beta=10$, the limit of the fitting function). The time evolution of the low energy spectral index $\alpha$ and of the peak energy $E_{\text {peak }}$ are reported in Fig. 3 (middle and bottom panels, respectively).

The hardness evolution of a burst is typically described by how the spectral index $\alpha$ and $E_{\text {peak }}=(\alpha+2) E_{0}$ change in time (here $E_{\text {peak }}$ is the peak energy of the $E F_{E}$ spectrum and $E_{0}$ is

\footnotetext{
${ }^{1}$ http://cossc.gsfc.nasa.gov/batse/4Bcatalog/ index.html
}

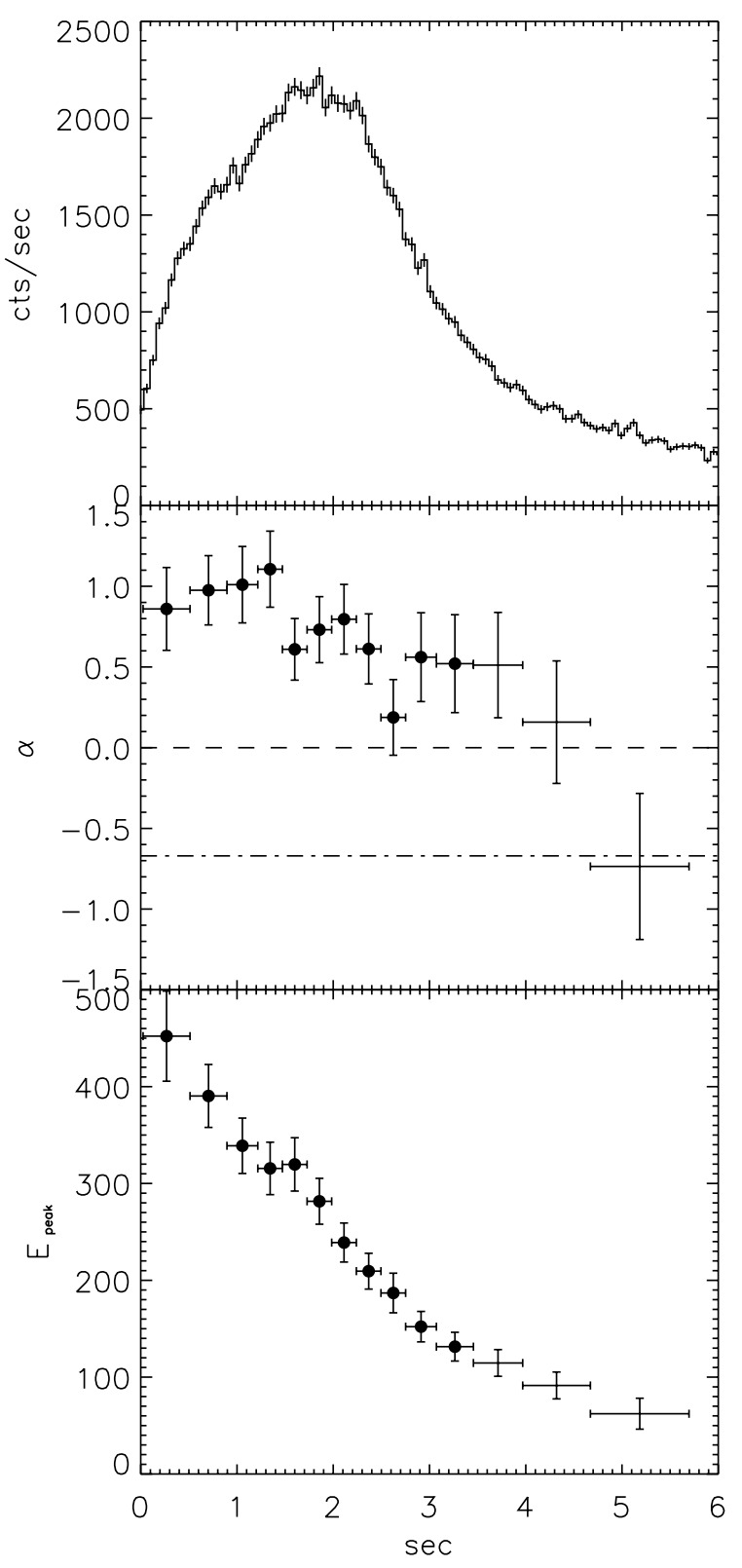

Fig. 3. Light curve and best fit parameters for GRB 980306. Top panel: light curve on the $64 \mathrm{~ms}$ timescale, integrated in the range 110$320 \mathrm{keV}$ and summed over all triggered detectors. Mid panel: low energy power law spectral index $(\alpha)$. Horizontal lines mark the limits $\alpha=-2 / 3$ (dot-dashed) and $\alpha=0$ (dashed). Bottom panel: peak energy $E_{\text {peak }}$ of the $E F_{E}$ spectrum. Error bars represent the $90 \%$ confidence intervals on the best fit parameters. The filled circles indicate the spectra which have been fitted also with a black body model (see Table 2 and Sect. 4.5).

the characteristic energy of the exponential cutoff). The evolution of $\alpha$ (mid panel of Fig. 3) indicates that, in the rising phase of the pulse, the maximum hardness $(\alpha=1.1 \pm 0.2)$ is reached during the interval $t \leq 1.5 \mathrm{~s}$. The first 4 spectra are in fact consistent with a non evolving spectrum (the low energy spectral index can be considered equal to its average value $\langle\alpha\rangle=0.73 \pm 0.03$ at $92 \%$ confidence level for the first $1.5 \mathrm{~s}$ ). After this time the spectrum softens, but it remains harder than $\alpha=0$. We stress that the low energy spectrum is significantly 
(more than $3 \sigma$ ) harder than $\alpha=0$ for each of the first 7 time resolved spectra (between $t=0$ and $t=2.24 \mathrm{~s}$ ). For this specific burst the evolution is hard-to-soft, as shown by the peak energy (bottom panel of Fig. 3) decreasing in time from $\sim 450 \mathrm{keV}$ to $\sim 50 \mathrm{keV}$, consistently with the $E_{\text {peak }}$ evolution reported by Ryde \& Svensson (2002).

In view of the discussion on the spectral models (Sect. 6) we also checked the inadequacy of the optically thin synchrotron model by fitting the BAND spectrum with the low energy power law slope fixed at $-2 / 3$. As expected, the residuals at low energies show a systematic sign and the reduced $\chi_{r}^{2}$ is larger than 2 indicating that this model is not a good representation of the data.

\subsection{GRB 911118}

The light curve of GRB 911118, reported in Fig. 4 (top panel), has two main peaks which partially overlap. It is a typical long burst with total duration $T_{90}=19.2 \pm 0.1 \mathrm{~s}$ and a background subtracted peak flux of $(30.6 \pm 0.8)$ phot $\mathrm{cm}^{-2} \mathrm{~s}^{-1}$ at $t_{\text {peak }}=$ $6.08 \mathrm{~s}$. Its fluence, in the $100-300 \mathrm{keV}$ energy range, is $(27.8 \pm$ $0.1) \times 10^{-6} \mathrm{erg} \mathrm{\textrm {cm } ^ { - 2 }}$.

The spectral analysis that we present covers the first $13 \mathrm{~s}$ after the trigger time (for a total of 50 spectra), in which the time resolved spectrum evolves dramatically with an excursion of $\Delta \alpha \sim 1$ and $\Delta E_{\text {peak }} \sim 400 \mathrm{keV}$. The best fit is obtained with the BAND model. The low energy spectral index is $\alpha>0$ for the first $\sim 6 \mathrm{~s}$ (32 spectra, phase A of Fig. 4, mid panel) and decreases with time (the spectrum softens), but remains harder than $-2 / 3$ for the time interval up to the end of phase B (see Fig. 4). After $t \sim 13 \mathrm{~s}$, the spectrum has a typical $\alpha<-2 / 3$ slope. The hardest spectrum (at $t \sim 1.5 \mathrm{~s}$ after the trigger) has $\alpha=0.74 \pm 0.13$. The statistical significance of a positive value of $\alpha$ for the spectra of phase A is high, being $>3 \sigma$ for most of the individual spectra (23/32). The peak energy evolution reported in Fig. 4 (bottom panel) is consistent with what reported by Ford et al. (1995). According to these fits, the 18 spectra belonging to phase $\mathrm{B}$ have $-2 / 3 \leq \alpha \leq 0$ and their evolution is of the "hard-to-soft" kind, i.e. the peak energy decreases in time (becoming smaller than $200 \mathrm{keV}$ in phase B). Figure 4 shows a possible correlation between the peak energy $E_{\text {peak }}$ and $\alpha$. It has been pointed out (Lloyd \& Petrosian 2000) that such correlations could result from the effect of the curvature of the fitted model when the peak energy is particularly low (e.g. below $\sim 100 \mathrm{keV}$ and close to the low energy spectral threshold). However in the case of GRB 911118 we can exclude this effect because the peak energy is between 500 and $200 \mathrm{keV}$ during phase A (Fig. 4).

Again, we also tried to fit the simplest optically thin synchrotron model (by fixing the low energy spectral index of the BAND model to -2/3). In Fig. 5 we report, for comparison, the values of the reduced $\chi_{r}^{2}$ for all spectra. The best fit is given by the BAND model (asterisks) while the "synchrotron" case (triangles) is inadequate to fit these spectra because its $\chi_{r}^{2}$ is typically $\geq 2$ (for $\sim 110$ d.o.f.) during phase A. The value of $\chi_{r}^{2}$ for the synchrotron model becomes marginally acceptable $(<1.5)$ for most spectra in phase B.

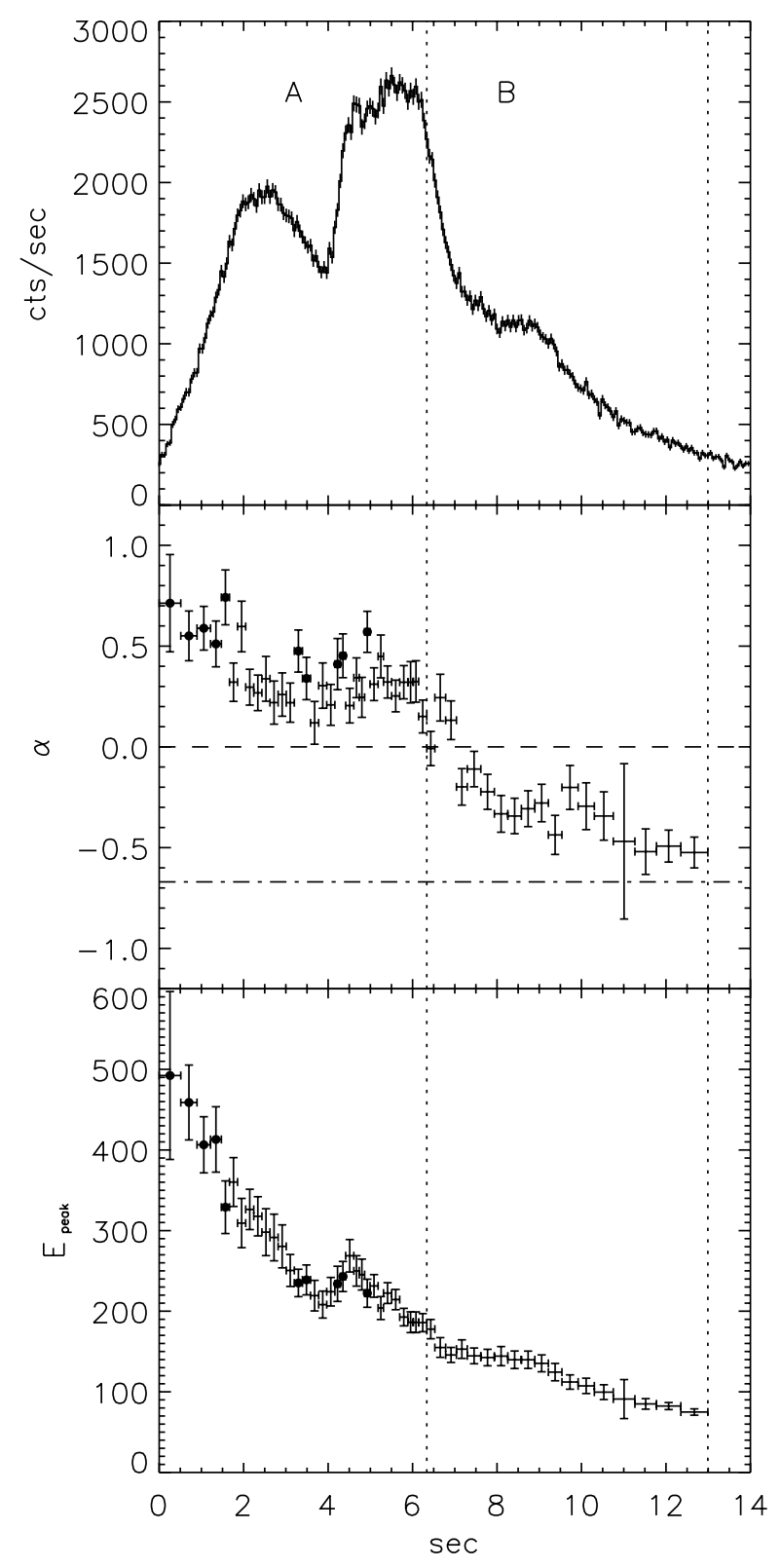

Fig. 4. The light curve and time evolution of the best fit parameters of the BAND model for GRB 911118. Top panel: light curve on the $64 \mathrm{~ms}$ timescale in the energy range 110-320 keV, summed over all the triggered detectors. The vertical dotted lines represent the boundaries of the phases in which the spectrum is harder than $E^{0}$ (phase A) and $E^{-2 / 3}$ (phase B). Mid panel: low energy power law spectral index $\alpha$, with the limits at $\alpha=-2 / 3($ dot-dashed $)$ and $\alpha=0$ (dashed). Bottom panel: peak energy of the $E F_{E}$ spectrum. The filled circles represent the spectra which have been fitted also with a blackbody model (see Table 2 and Sect. 4.5).

We conclude that the time resolved spectra of this burst are best represented by the BAND model with the 4 fit parameters free to vary and that most of the spectra of phase A have $\alpha>0$ at $\mathrm{a} \geq 3 \sigma$ level. 


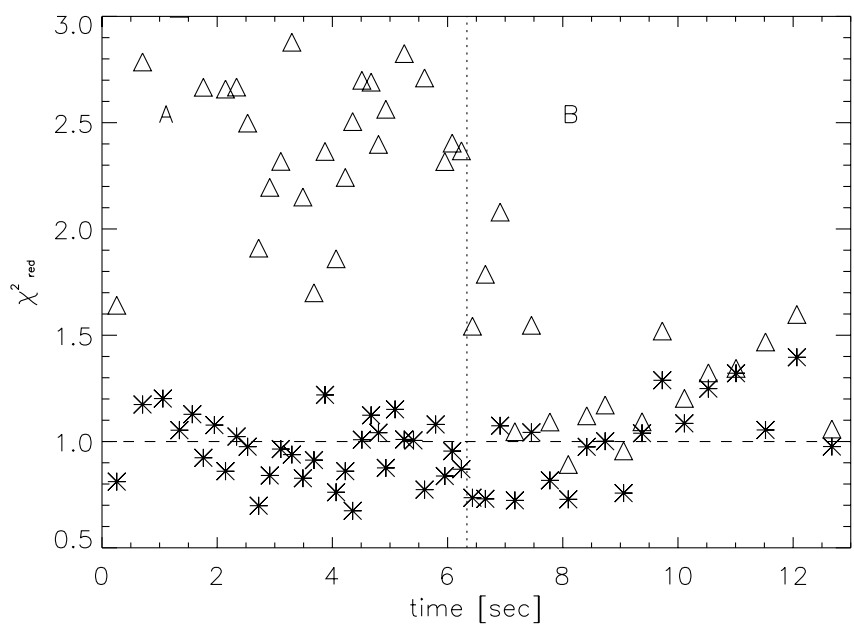

Fig. 5. Reduced $\chi_{r}^{2}$ (for typical 110 d.o.f.) of the fits with different models to the spectra of GRB 911118: BAND model (asterisks), BAND model with the low energy spectral index fixed at $-2 / 3$ (triangles).

\subsection{Tests on the spectral results}

Considering the relevance of the extreme hardness of these bursts for the comparison with the models, we performed different checks to verify if and at what confidence level they can be considered harder than the predictions of the proposed spectral models (as in Table 1). In the following we report on the tests performed on the above GRB spectra and their results.

- Reduced fitting energy range: we eliminated from the fitting energy range the high energy channels to test if they substantially influence the low energy fitted spectral slope. We restricted the analysis to the range $28-600 \mathrm{keV}$ (the upper limit being the maximum peak energy found among the bursts presented) and compared the results with those obtained by fitting the spectra on the entire energy window (i.e. up to $1800 \mathrm{keV}$ ). We found that the best fit spectral parameters are consistent within their errors and the values of $\alpha$ inferred from the limited energy range case are still inconsistent with $\alpha=0$. Moreover, Preece et al. (1998) found a soft low energy component in some bursts through the analysis of other data type extending below the standard LAD threshold of $28 \mathrm{keV}$. This component could make the fitted low energy spectral slope softer than it really is and the fit results for the $\alpha$ parameter presented here should be considered as lower limits thus reinforcing the evidence of extremely hard spectra. We tested this possibility by fitting the spectra with a low energy threshold at 50 and $100 \mathrm{keV}$. The comparison with the fits on the entire energy range (28-1800 keV) showed consistent spectral parameters within their $90 \%$ confidence intervals.

- Background spectrum: if the background spectrum were characterized by any feature or absorption edge at low energies (e.g. dependent on some electronic failure or anomalous observational conditions) this might determine particularly hard low energy power laws in the background subtracted spectra. In order to exclude this possibility we computed several backgrounds selecting different time intervals, within the same GRB, and found that the background spectrum does not depend on the time interval selected for its estimate. Moreover, we also fitted the spectra adopting different backgrounds calculated for other bursts: the results indicate that $\alpha$ is not affected by anomalous features in the background spectrum.

- Detector response: the detector response matrix, if incorrectly calculated, could determine a wrong set of best fit parameters. We believe this is unlikely as, by fitting the spectra with the detector response matrix (DRM) associated with another burst but for the same LAD, the results are consistent within their $90 \%$ confidence level. Moreover, although we used the data of the mostly illuminated detector, we also tried to fit the data from the other three detectors that were triggered by these bursts. Although the lower signal to noise ratio in these "secondary" detectors resulted in a lower statistical significance of the best fit parameters, these fits confirmed the extremely hard low energy spectral slope of these bursts at least in the second best illuminated detector. For the remaining two detectors the results are consistent, although the low statistics does not allow to confirm the hardness. Note also that Crider et al. (1997) showed that combining different detectors can make the low energy spectral component softer than it really is.

- Comparison with a simulated spectrum: the most direct and robust way to verify if the deconvolved spectra are really as hard as we found is to compare them with a reference spectrum whose shape is well defined. For every time resolved spectrum that we analyzed, we thus simulated a reference (template) spectrum with a low energy power law slope $\alpha \sim 0$ and a break energy fixed at the value obtained from the fit of the observed spectrum. We divided channel by channel the observed spectrum by the template and then analyzed the ratio (as long as the observed spectrum is harder than the template one their ratio at low energies increases with energy). In order to quantify the hardness we then fitted such ratio $R(E)$, where $E$ represents the energy, with a power-law model $R(E)=A E^{\delta}+B$. The fitted index $\delta$ is reported in Figs. 6 and 7 for GRB 911118 and GRB 980306, respectively. We note that during phase A (the first $6 \mathrm{~s}$ after the trigger) of GRB 911118 each of the time resolved spectra is at least $3 \sigma$ harder than a flat spectrum (i.e. with the same level of confidence that was found with the direct fitting method). In the case of GRB 980306 this test gives a fitted ratio with a poor level of confidence $(<2 \sigma)$ for each spectrum (with only two spectra at $>2 \sigma$ ) because of the lower $\mathrm{S} / \mathrm{N}$ due to count statistics and to the propagation of errors in dividing the observed spectrum by the template one. This significance is increased if the first 8 spectra are rebinned in time (although the time resolved information is lost) which gives a value of $\delta>0$ at $3.7 \sigma$. Furthermore (see also below) also in the case of GRB 980306 the significance of $\delta>0$ (Fig. 7) is higher when considering the sequence of $\delta$ values as a group (since $\delta$ remains systematically positive).

As a final consideration let us stress the fact that in the case of GRB 911118 (Fig. 4) and GRB 980306 (Fig. 3) there is 


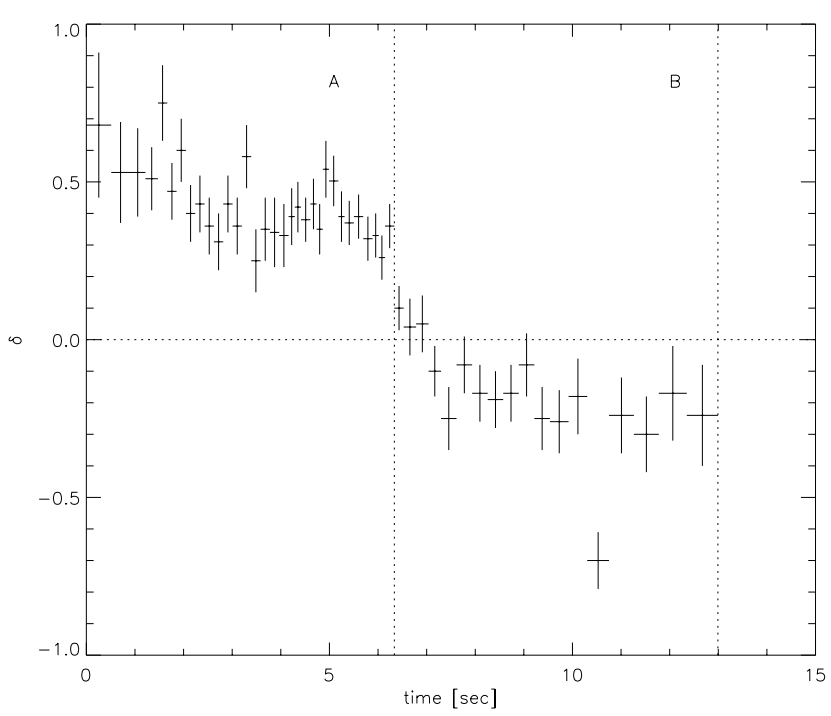

Fig. 6. GRB 911118 - slope of the power law fitted to the ratio between the observed time resolved spectra and the template spectra (simulated with $\alpha=0$ and $E_{\text {break }}=E_{0}(f i t)$ ). Labels A and B refer to the two phases of GRB 911118 (Fig. 4) and the horizontal dotted line is the slope of the reference spectrum.

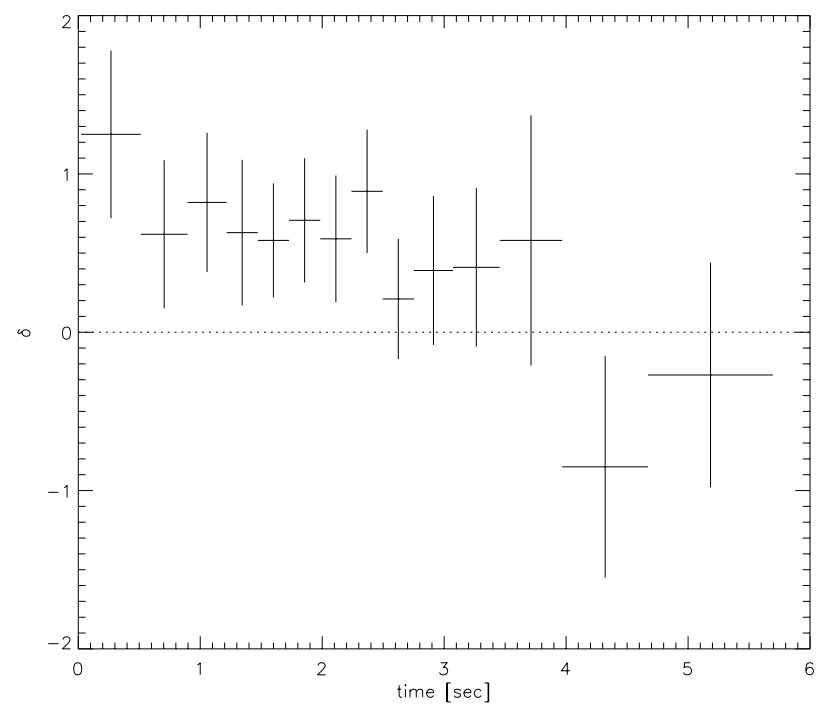

Fig. 7. As Fig. 6 for GRB 980306.

a minority of hard spectra ( 9 and 4 , respectively) which have $\alpha>0$ at a level of significance lower than $3 \sigma$. Nonetheless, the fact that a considerable number of subsequent spectra (32 for GRB 911118 and 12 for GRB 980306) in the first few seconds have a spectrum harder (at more than $3 \sigma$ ) than $E^{0}$ increases the level of significance of the result, if they are considered as a whole.

\subsection{Other hard bursts}

There are other bursts with extremely hard low energy spectra for a major part of their evolution, which have already been reported in the literature. In particular, we found three bursts with very hard spectra if fitted by the BAND function. In order to have homogeneous results and verify their robustness we have

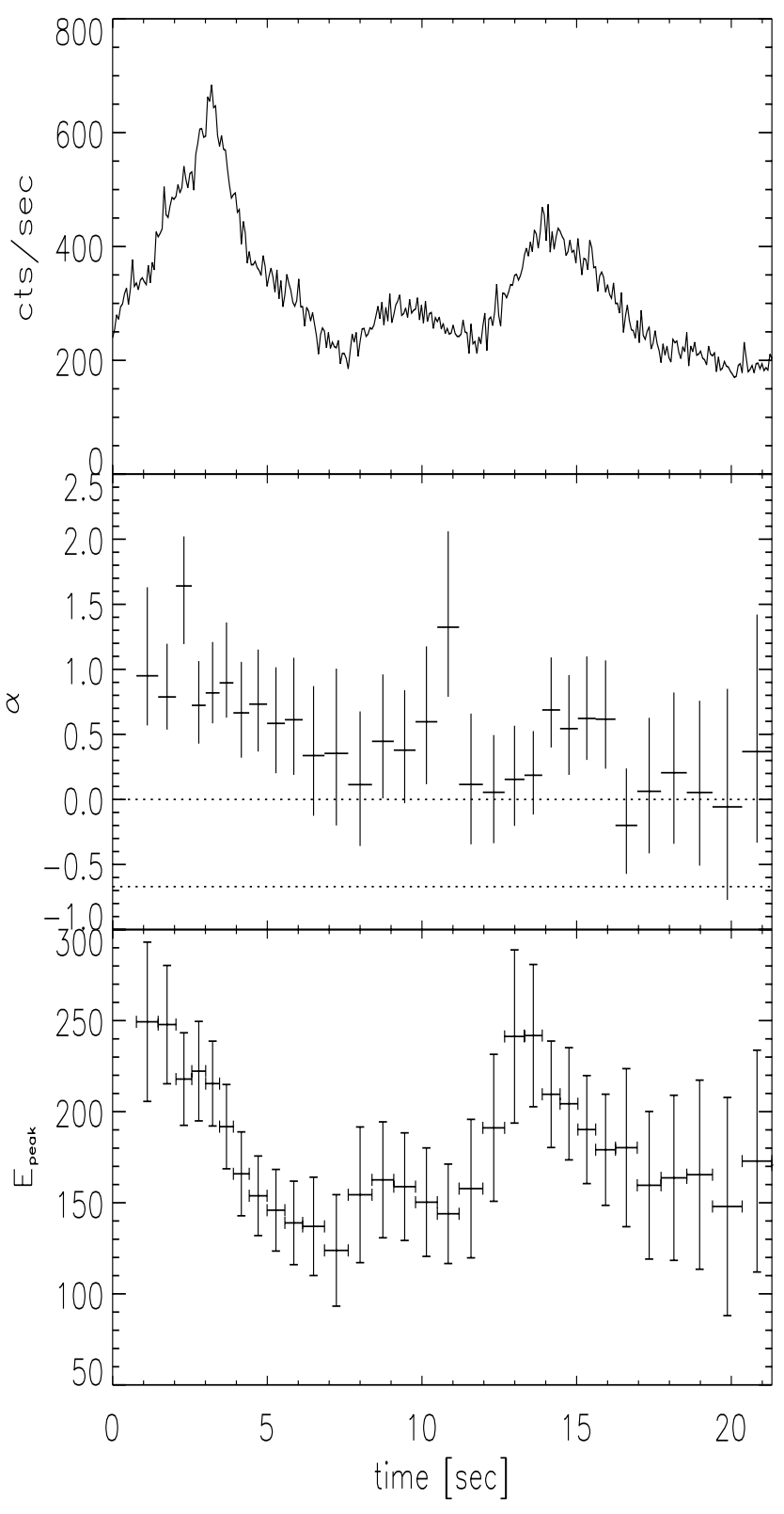

Fig. 8. GRB 910807 spectral evolution. Same as Fig. 3.

then re-analyzed their spectra and applied the tests described in Sect. 4.3. Let us summarize their properties.

- GRB 910807 has a peak flux of $(7.2 \pm 0.5)$ phot $\mathrm{cm}^{-2} \mathrm{~s}^{-1}$. The spectrum is harder than $\alpha=0$ for the first $\sim 5 \mathrm{~s}$, and remains quite constant $(\sim 0)$ during the overall burst, which lasts about $28 \mathrm{~s}$, as already pointed out by Ryde \& Svensson (1999). The peak energy instead shows a tracking pattern correlated with the flux and hardens in correspondence of the second peak (at $t=12 \mathrm{~s}$ ) as already reported by Borgonovo \& Ryde (2001).

The complete spectral evolution is reported in Fig. 8. The hardest spectrum has $\alpha=1.6 \pm 0.5$ at $90 \%$ confidence level. This value is somewhat harder, but consistent with what quoted by Crider et al. (2000). A (successful) check for the hardness of the first few spectra of this burst has been 
performed using the last method described in the previous section.

- GRB 910927 (Crider et al. 2000, 1997b) and GRB 970111 (Crider et al. 1997a; Frontera et al. 2000) are other two cases of extremely hard spectra with a low energy power law spectral index as high as $\alpha=1.6 \pm 0.3$. GRB 970111 was studied (Frontera et al. 2000) also including the WFCBeppoSAX data. Its extremely hard low energy spectrum was confirmed to extend down to $5 \mathrm{keV}$. Unfortunately, the lack of spectral resolution of the GRBM instrument above $40 \mathrm{keV}$ does not allow to study the complete spectrum, especially at its peak.

In addition we found in the spectral catalogue of Preece et al. (2000) other 3 bursts (BATSE triggers 1974, 2855 and 6350) with $\alpha>0$, but at a low significance level $(<1 \sigma)$ and/or for a short interval of the light curve. Note that also these cases contribute to the $\alpha>0$ tail of the spectral index distribution reported in Fig. 1.

\subsection{Black body spectra}

As anticipated we performed fits on the hardest spectra also with a black body function to test if the emission is consistent with a thermal model. In fact, the first spectra of all the bursts discussed in this work are typically harder than $\alpha=0.5$ and thus could be consistent with the Rayleigh-Jeans part of a black body. Note that the fit could however be unacceptable in terms of $\chi_{r}^{2}$ if the curvature around the peak (i.e. typically in the range $100-300 \mathrm{keV}$ ) were broader than a black body one.

As already mentioned the fits have been performed only on spectra with $\alpha \gtrsim 0.5$. In order to determine the value of this threshold in $\alpha$ we simulated spectra with a black body model assuming the detector response of these bursts and a typical exposure time $\sim 0.2 \mathrm{~s}$ (i.e. the average integration time of the spectra presented before). We then fitted these simulated thermal spectra with a non-thermal model $N(E) \propto E^{\alpha} \exp \left(-E / E_{0}\right)$ where the power law spectral index $\alpha$ was fixed at different values between 0 (a flat photon spectrum) and +1 (which best approximates a thermal spectrum). The residuals of these fits indicated that for $\alpha<0.5$ the model spectrum (at low energies) deviates systematically from the data (i.e. residuals different from 0 ) at more than $2 \sigma$. For this reason we selected $\alpha=0.5$ as the threshold for fitting the spectra with a black body model.

The criteria applied for the goodness of the fit with the black body model were:

- The reduced $\chi_{\mathrm{r}}^{2}$ should be smaller than a fixed reference value of 1.5 .

- The data-to-model ratio can be systematically and significantly greater than 1 only at energies above the peak, as a possible consequence of the presence of a supra-thermal or multi-temperature spectral component (whose nature is not discussed here).

The results of the fits for each spectrum are reported in Table 2: the start and stop time, with respect to the trigger time, are given in Cols. 2 and 3. The model temperature $k T(\mathrm{keV})$ and

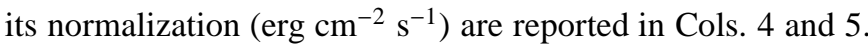
The value of the reduced $\chi_{r}^{2} /$ d.o.f. with the corresponding degrees of freedom and the photon flux computed from the fitted model in the $28-1800 \mathrm{keV}$ energy range are given in Cols. 6 and 7 , respectively. Columns 8 and 9 report the spectral index $(\alpha)$ obtained by the fit with the non thermal (BAND or COMP) models and the associated $\chi_{r}^{2} /$ d.o.f.

For illustration, we report in Fig. 9 some examples of fitted spectra for GRB 980306 and the corresponding data-to-model ratios. The first two spectra are considered well fitted by the model because $\chi_{r}^{2}=1.19$ and 0.9 for 111 d.o.f., respectively, and the ratio is systematically greater than 1 only at high energies. In the other two cases the black body fits give less acceptable results because the data-to-model ratio shows systematic deviations from 1 at low energies.

In most of the bursts the best fits with the black body model are obtained at the beginning: in the later stages (e.g. after the first $2.5 \mathrm{~s}$ in the case of GRB 980306) the low energy component softens and becomes incompatible with the black body model as the reduced $\chi_{r}^{2}$ and the data-to-model ratio indicate.

The black body temperature and total flux (as derived by the model) evolve in time as reported in Fig. 10 for all the good black body spectral fits. Note the decrease of the temperature with time in almost all these bursts, which in the figure are compared with a dependence $T_{\mathrm{BB}} \propto t^{-1 / 4}$ (dotted line). Although the temperature decreases, the flux slightly increases or remains constant in the first phases, and rapidly decreases thereafter.

Before concluding we note that although not publicly available, other possible evidence of a black body emission from GRBs has been reported in poster proceedings (Palmer, private communication). The possible thermal black body spectrum of GRB970111 has also been recently presented by Preece (2001): the spectrum integrated over its first $5 \mathrm{~s}$ could be fitted by a black body with $k T=55 \mathrm{keV}$ which is consistent with the average value of the black body temperatures that we obtain from the fits of the time resolved spectra covering the same time interval (Fig. 10).

\section{Discussion}

GRB 980306 and 911118, together with GRB910807, 910927 and 970111 already reported in the literature, bring new constraints on the extremely hard spectra that the emission process responsible for the prompt phase must be able to satisfy. These bursts represent the cases with the hardest low energy power law spectral index and significantly contribute in extending the $\alpha$ distribution reported by Preece at al. (2000) and Ghirlanda et al. (2002) to values greater than 0, up to $\alpha \sim 1.5$. For the bursts studied here $\alpha$, i.e. the spectral index of the low energy photon spectrum, remains positive for a major part of the pulse/s, like in the case of GRB 911118 or GRB 970111, or for its first rising part (GRB 980306 and GRB 910807).

In the following we consider several emission models proposed so far to see if they can account for these hard spectra, except for the photospheric and Compton drag model which will be discussed in Sect. 5.5.2 in relation to the quasi-thermal character of the initial phases of these extremely hard bursts. 
Table 2. Black body fits.

\begin{tabular}{|c|c|c|c|c|c|c|c|c|}
\hline GRB & $\begin{array}{c}t_{\text {start }} \\
\mathrm{s}\end{array}$ & $\begin{array}{c}t_{\text {stop }} \\
\mathrm{s}\end{array}$ & $\begin{array}{c}k T \\
\mathrm{keV}\end{array}$ & $\begin{array}{c}N \\
\mathrm{erg} / \mathrm{cm}^{2} \mathrm{~s}\end{array}$ & $\chi_{r}^{2}$ (d.o.f.) & $\begin{array}{c}F \\
\text { phot } / \mathrm{cm}^{2} \mathrm{~s}\end{array}$ & $\alpha$ & $\chi_{r, \alpha}^{2}$ (d.o.f.) \\
\hline \multirow[t]{11}{*}{980306} & 0.024 & 0.512 & $104_{-4.8}^{+4.8}$ & $46_{-2.8}^{+2.7}$ & $1.15(107)$ & 8.4 & $0.86 \pm 0.15$ & $1.09(109)$ \\
\hline & 0.512 & 0.896 & $93_{-3.4}^{+4.6}$ & $63_{-2.8}^{+3.2}$ & $1.27(107)$ & 12.9 & $0.98 \pm 0.13$ & $1.21(104)$ \\
\hline & 0.896 & 1.216 & $81_{-2.8}^{+2.9}$ & $63_{-2.8}^{+2.8}$ & $1.27(107)$ & 14.7 & $1.01 \pm 0.13$ & $1.2(106)$ \\
\hline & 1.216 & 1.472 & $78_{-2.6}^{+2.8}$ & $68_{-3.0}^{+3.1 .}$ & $1.19(103)$ & 16.6 & $1.11 \pm 0.14$ & $1.19(102)$ \\
\hline & 1.472 & 1.728 & $72_{-2.5}^{+2.5}$ & $\begin{array}{l}70_{-3.0}^{+3.0} \\
\end{array}$ & $1.44(105)$ & 18.4 & $0.61 \pm 0.12$ & $1.12(104)$ \\
\hline & 1.728 & 1.984 & $65_{-2.1}^{+2.1}$ & $66_{-2.6}^{+3.0}$ & $0.86(107)$ & 19.10 & $0.73 \pm 0.12$ & $0.7(106)$ \\
\hline & 1.984 & 2.24 & $57_{-1.8}^{+1.6}$ & $59_{-2.6}^{+1.0}$ & $1.33(104)$ & 19.09 & $0.8 \pm 0.13$ & $1.16(103)$ \\
\hline & 2.24 & 2.496 & $49_{-1.4}^{+1.8}$ & $52_{-1.8}^{+1.8}$ & $1.26(104)$ & 19.3 & $0.61 \pm 0.13$ & $0.96(103)$ \\
\hline & 2.496 & 2.752 & $42_{-1.4}^{-1.4}$ & $39_{-1.4}^{+1.8}$ & $1.39(101)$ & 16.6 & $0.19 \pm 0.14$ & $0.8(100)$ \\
\hline & 2.752 & 3.072 & $37_{-1.1}^{-1.4}$ & $30_{-0.9}^{-1.4}$ & $1.45(104)$ & 14.1 & $0.56 \pm 0.17$ & $1.2(103)$ \\
\hline & 3.072 & 3.456 & $33_{-1.0}^{+1.0}$ & $23_{-0.8}^{+0.7}$ & $1.23(107)$ & 12.14 & $0.52 \pm 0.19$ & $1.0(106)$ \\
\hline \multirow[t]{7}{*}{911118} & 0.000 & 0.512 & $114_{-8.0}^{+8.0}$ & $23_{-3.0}^{+2.0}$ & $0.95(111)$ & 3.92 & $0.71 \pm 0.24$ & $0.8(109)$ \\
\hline & 0.512 & 0.896 & $98_{-5.0}^{+4.0}$ & $39_{-3.0}^{+2.0}$ & $1.39(109)$ & 7.61 & $0.55 \pm 0.12$ & $1.1(107)$ \\
\hline & 0.896 & 1.216 & $\begin{array}{l}87_{-5.0}^{+3.0} \\
\text { 3. }\end{array}$ & $52_{-3.0}^{+3.0}$ & $1.51(105)$ & 11.28 & $0.59 \pm 0.11$ & $1.2(103)$ \\
\hline & 1.216 & 1.472 & $89_{-4.0}^{-5.0}$ & $69_{-4.0}^{+4.0}$ & $1.45(107)$ & 14.78 & $0.51 \pm 0.11$ & $1.0(105)$ \\
\hline & 1.472 & 1.664 & $76_{-3.0}^{-4.0}$ & $68_{-3.0}^{+4.0}$ & $1.35(105)$ & 16.93 & $0.74 \pm 0.13$ & 1.1(103) \\
\hline & 3.2 & 3.392 & $\begin{array}{l}52_{-2.0}^{+1.0} \\
\end{array}$ & $65_{-2.0}^{+2.0}$ & $1.52(104)$ & 22.76 & $0.5 \pm 0.1$ & $0.9(102)$ \\
\hline & 3.392 & 3.584 & $\begin{array}{r}52_{-2.0}^{+1.3} \\
\text { 1. }\end{array}$ & $\begin{array}{l}59_{-2.0}^{+2.0} \\
\text { - }\end{array}$ & $1.51(107)$ & 20.61 & $0.57 \pm 0.1$ & $0.87(105)$ \\
\hline \multirow[t]{15}{*}{910807} & 0.768 & 1.472 & $71_{-4.6}^{+4.6}$ & $9.4_{-0.8}^{+0.8}$ & $1.10(113)$ & 2.51 & $0.95 \pm 0.29$ & $1.2(111)$ \\
\hline & 1.472 & 2.048 & $61_{-3.5}^{-4.3 .3}$ & $11_{-0.9}^{-0.8}$ & $1.29(113)$ & 3.3 & $0.78 \pm 0.21$ & $1.0(111)$ \\
\hline & 2.048 & 2.56 & $59_{-26}^{+2.8}$ & $\begin{array}{r}-0.9 \\
17_{-0.8}^{+0.8}\end{array}$ & $1.05(112)$ & 5.32 & $1.64 \pm 0.25$ & $0.8(110)$ \\
\hline & 2.56 & 3.008 & $57_{-2.3}^{+2.2}$ & $\begin{array}{l}19_{-2.3}^{+1.2} \\
\end{array}$ & $0.78(111)$ & 6.12 & $0.72 \pm 0.19$ & $0.84(109)$ \\
\hline & 3.008 & 3.456 & $53_{-2.1}^{+2.2}$ & $21_{-0.9}^{+0.9}$ & $0.94(109)$ & 7.36 & $0.82 \pm 0.18$ & $0.95(107)$ \\
\hline & 3.456 & 3.904 & $53_{-1.9}^{+1.1}$ & $24_{-1.0}^{+1.9}$ & $1.00(112)$ & 8.43 & $0.89 \pm 0.21$ & $1.06(110)$ \\
\hline & 3.904 & 4.416 & $47_{-1.7}^{-1.9}$ & $\begin{array}{r}-1.0 \\
19_{-0.9}^{+0.9}\end{array}$ & $1.09(111)$ & 7.42 & $0.67 \pm 0.23$ & $0.97(109)$ \\
\hline & 4.416 & 4.992 & $41_{-1.7}^{+1.6}$ & $14_{-0.6}^{+0.6}$ & $1.06(113)$ & 6.05 & $0.58 \pm 0.25$ & $1.0(111)$ \\
\hline & 4.992 & 5.568 & $38_{-1.6}^{+1.1 .5}$ & $11_{-0.5}^{+0.0}$ & $0.84(112)$ & 5.39 & $0.61 \pm 0.28$ & $1.1(110)$ \\
\hline & 5.568 & 6.144 & $36_{-1.5}^{+1.5}$ & $11_{-0.5}^{-0.5}$ & $1.11(112)$ & 5.23 & $0.6 \pm 0.33$ & $1.17(110)$ \\
\hline & 9.792 & 10.496 & $39_{-1.9}^{-1.0}$ & $7.7_{-0.4}^{-0.4}$ & $1.24(113)$ & 3.56 & $1.32 \pm 0.40$ & $1.63(111)$ \\
\hline & 10.496 & 11.2 & $37_{-2.0}^{-1.9}$ & $6.8_{-0.4}^{-0.4}$ & $1.21(114)$ & 3.2 & $0.54 \pm 0.23$ & $1.03(112)$ \\
\hline & 14.464 & 15.04 & $50_{-2.2}^{+2.3 .3}$ & $14_{-0.8}^{-0.4}$ & $0.83(112)$ & 5.16 & $0.62 \pm 0.24$ & $0.83(110)$ \\
\hline & 15.04 & 15.616 & $49_{-2.2}^{+2.2 .2}$ & $13_{-0.7}^{+0.8}$ & $1.11(115)$ & 4.851 & $0.62 \pm 0.27$ & $1.4 / 109$ \\
\hline & 15.616 & 16.256 & $46_{-2.2}^{+2.2}$ & $12_{-0.7}^{+0.6}$ & $0.9(112)$ & 4.6 & $0.61 \pm 0.27$ & $1.4(109)$ \\
\hline \multirow[t]{9}{*}{910927} & 0.000 & 0.704 & $63_{-3.8}^{+3.8}$ & $7_{-0.5}^{+0.6}$ & $0.82(107)$ & 2.26 & $0.62 \pm 0.26$ & $0.96(105)$ \\
\hline & 0.704 & 1.28 & $53_{-2.4}^{+3.8}$ & $12_{-0.6}^{-0.5}$ & $0.91(109)$ & 4.05 & $1.02 \pm 0.28$ & $0.93(107)$ \\
\hline & 1.28 & 1.792 & $46_{-1.7}^{+2.4}$ & $12_{-0.6}^{+0.6}$ & $1.3(107)$ & 4.9 & $1.04 \pm 0.25$ & $1.13(105)$ \\
\hline & 1.792 & 2.304 & $42_{-1.3}^{+1.1}$ & $12_{-0.6}^{+0.0}$ & $1.23(105)$ & 5.35 & $1.24 \pm 0.24$ & $0.9(103)$ \\
\hline & 2.304 & 2.752 & $41_{-1.4}^{+1.3}$ & $14_{-0.6}^{-0.0}+0.6$ & $0.99(104)$ & 5.9 & $1.1 \pm 0.24$ & $0.8(102)$ \\
\hline & 2.752 & 3.2 & $37_{-1.2}^{-1.4}$ & $15_{-0.5}^{+0.6}$ & $0.98(105)$ & 6.9 & $1.05 \pm 0.24$ & $1.0(103)$ \\
\hline & 3.2 & 3.584 & $37_{-1.2}^{-1.2}$ & $17_{-0.7}^{-0.6}$ & $0.98(101)$ & 7.85 & $1.12 \pm 0.25$ & $1.0(99)$ \\
\hline & 3.584 & 3.968 & $36_{-1.0}^{+1.0}$ & $17_{-0.6}^{+0.6}$ & $0.99(101)$ & 8.03 & $0.98 \pm 0.19$ & $1.1(99)$ \\
\hline & 3.968 & 4.416 & $32_{-1.0}^{+1.0}$ & $13_{-0.5}^{+0.5}$ & $0.8(105)$ & 6.9 & $0.83 \pm 0.2$ & $0.8(103)$ \\
\hline \multirow[t]{12}{*}{970111} & 0.576 & 1.088 & $61_{-2.0}^{+2.0}$ & $11_{-0.8}^{+0.8}$ & $0.97(108)$ & 3.2 & $1.51 \pm 0.4$ & $0.96(106)$ \\
\hline & 1.088 & 1.6 & $56_{-2.3}^{+2.3}$ & $\begin{array}{l}-0.8 \\
10_{-0.6}^{+0.6}\end{array}$ & $1.15(105)$ & 3.23 & $1.31 \pm 0.34$ & $1.07(103)$ \\
\hline & 1.6 & 2.112 & $51_{-2.3}^{-2.3 .3}$ & $9.3_{-0.6}^{+0.6}$ & $1.00(108)$ & 3.3 & $1.11 \pm 0.28$ & $0.9(106)$ \\
\hline & 2.112 & 2.58 & $49_{-2.4}^{+2.3}$ & $\begin{array}{l}9_{-0.6}^{+0.6} \\
\text { +0.6 }\end{array}$ & $1.43(105)$ & 3.32 & $1.02 \pm 0.31$ & $1.2(103)$ \\
\hline & 2.58 & 3.008 & $48_{-2.4}^{+2.4}$ & $\begin{array}{l}-0.0 \\
9_{-0.6}^{+0.6}\end{array}$ & $0.98(104)$ & 3.3 & $1.6 \pm 0.4$ & $0.9(102)$ \\
\hline & 3.008 & 3.456 & $49_{-2.4}^{-2.4}$ & $10_{-0.6}^{+0.6}$ & $0.93(104)$ & 3.6 & $1.02 \pm 0.32$ & $0.8(102)$ \\
\hline & 3.456 & 3.904 & $46_{-2.1}^{-2.4}$ & $10_{-0.5}^{-0.5}$ & $0.97(102)$ & 4.0 & $1.85 \pm 0.38$ & $0.7(100)$ \\
\hline & 3.904 & 4.288 & $46_{-2.2}^{+2.1}$ & $10_{-0.06}^{+0.5}$ & $0.95(104)$ & 4.06 & $1.83 \pm 0.4$ & $0.9(102)$ \\
\hline & 4.288 & 4.672 & $40_{-1.4}^{+1.9}$ & $9.7_{-0.6}^{-0.6}$ & $1.16(100)$ & 4.27 & $1.25 \pm 0.31$ & $1.2(98)$ \\
\hline & 4.672 & 5.056 & $42_{-2.0}^{-1.4}$ & $10_{-0.6}^{+0.6}$ & $1.13(98)$ & 4.3 & $1.34 \pm 0.41$ & $0.92(96)$ \\
\hline & 5.056 & 5.44 & $43_{-2.0}^{+2.0}$ & $\begin{array}{r}-0.6 \\
11_{-0.7}^{+0.7}\end{array}$ & $0.96(104)$ & 4.7 & $0.79 \pm 0.31$ & $1.07(102)$ \\
\hline & 5.44 & 5.76 & $44_{-2.0}^{+2.0}$ & $12_{-0.7}^{+0.7}$ & $0.85(101)$ & 5.0 & $0.84 \pm 0.28$ & $0.82(99)$ \\
\hline
\end{tabular}



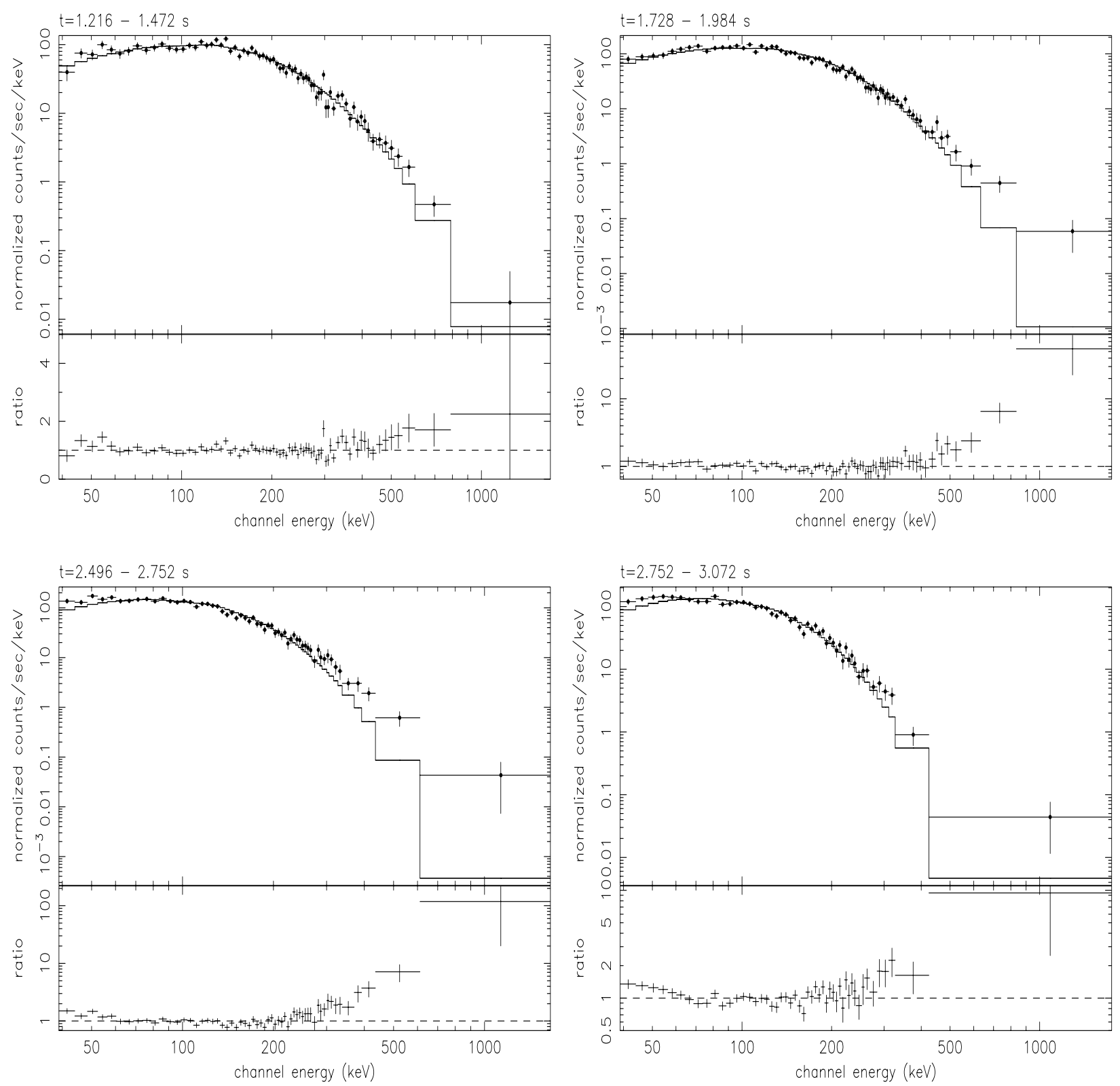

Fig. 9. Examples of black body fits for GRB 980306 (see Table 2). The integration times are indicated for each plot. The data-to-model ratios are reported in the bottom panels.

\subsection{Synchrotron emission}

Optically thin synchrotron emission - The optically thin synchrotron model, in its simplest formulation, can produce at most a spectrum as hard as $\alpha=-2 / 3$ (Katz 1994). Furthermore if one considers the very short cooling timescales (much shorter than any conceivable current exposure time) the predicted "cooled" spectrum has $\alpha=-3 / 2$ (see also Ghisellini et al. 2000).

Synchrotron self-absorption - One alternative, investigated among others by Papathanassiou (1999), is that the medium responsible for the synchrotron emission also absorbs these photons. Also Granot et al. (2000) have proposed a model based on synchrotron self absorption by a stratified electron population. In these cases it is possible to produce low energy spectral slopes as hard as $\alpha=1$ (for a thermal or a power law electron energy distribution with a low energy cut-off) or $\alpha=1.5$ (for a power law extending to low energies).

However, for synchrotron self-absorption to be effective in the typical BATSE energy range, a very high density of relativistic electrons is necessary. Assuming that the electron distribution extends to low energies (this minimizes the required particle number) with a power law distribution of index $p$ (i.e. $N_{\mathrm{e}}(\gamma) \propto \gamma^{-p}$, where $\gamma$ is the Lorentz factor of the electrons), the synchrotron self-absorption frequency $v_{\mathrm{t}}$ in the comoving frame, assuming $p=2$, is (e.g. Ghisellini \& Svensson 1991) 


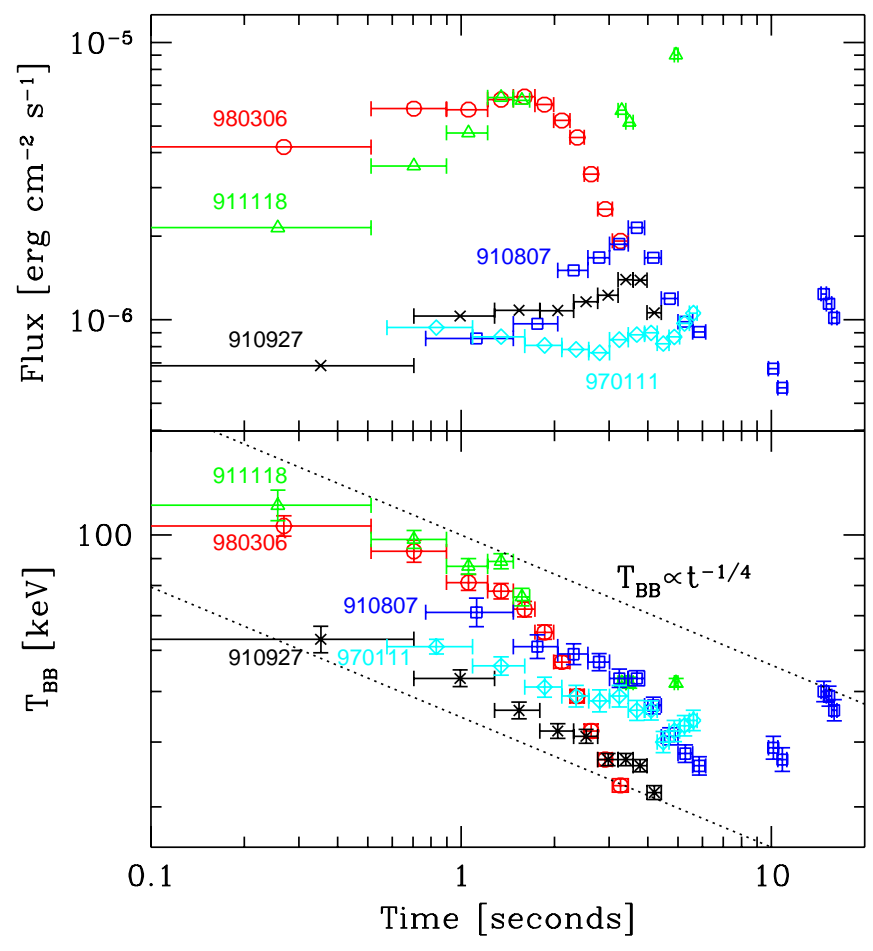

Fig. 10. Total flux (top panel) and black body temperature (bottom panel) as a function of time for the spectra reported in Table 2 and considered acceptable in terms of residuals. The dashed lines in the bottom panel correspond to $T_{\mathrm{BB}} \propto t^{-1 / 4}$.

$v_{\mathrm{t}} \simeq 2 \times 10^{5} v_{\mathrm{B}}\left(\tau_{\mathrm{T}} / B\right)^{1 / 3} \mathrm{~Hz}$, where $\tau_{\mathrm{T}}$ is the Thomson optical depth and $v_{\mathrm{B}}=e B /\left(2 \pi m_{\mathrm{e}} c\right)$ is the Larmor frequency. In order to have $v_{\mathrm{t}} \sim 10^{16} \mathrm{~Hz}$, corresponding to $\sim \Gamma v_{\mathrm{t}}$ in the BATSE range, $\tau_{\mathrm{T}} B^{2} \sim 5.7 \times 10^{12} v_{\mathrm{t}, 16}^{3}$. The Comptonization parameter $y \sim \tau_{\mathrm{T}}\left\langle\gamma^{2}\right\rangle$ corresponding to the required particle density, would largely exceed unity (making inverse Compton process more efficient than synchrotron) unless the magnetic field is extremely high $\left(B \sim 10^{7} \mathrm{G}\right)$. This would in turn imply a (magnetically dominated) fireball with isotropic (equivalent) energy in excess of $10^{54} \mathrm{erg}$, using $E \sim 4 \pi R^{2}\left(B^{2} / 8 \pi\right) \Gamma^{2} c t_{\text {burst }}$ and assuming $\Gamma \sim 10^{2}$ and $R \sim 10^{13} \mathrm{~cm}$. A magnetic field of $\sim 10^{7} \mathrm{G}$ has been derived also by Crider \& Liang (1999) from the fit of a simplified self absorbed synchrotron model to the spectrum of GRB 970111.

Small pitch angles - Lloyd \& Petrosian (2000) have proposed that in rarefied, highly magnetized plasmas, turbulence can give origin to an anisotropic pitch angle distribution of emitting particles. In this case the optically thin synchrotron spectrum is modified at low energies (compared to the standard $\left.F(E) \propto E^{1 / 3}\right)$, with a limiting slope $F(E) \propto E$. This can accommodate some, but not all, of the hard spectra that we presented. Note also that according to this scenario to prevent strong cooling the particles have to be re-accelerated, contrary to one of the basic assumptions of the internal shock scenario, and in order not to be re-isotropized by scattering, the inverse Compton process must be much less efficient than the synchrotron one.

\subsection{Jitter radiation}

A variant of the standard synchrotron emission theory, the jitter radiation (Medvedev 2001), can justify a flat low energy spectral slope. It originates as the emission of relativistic electrons in a non uniform magnetic field with inhomogeneity length scale smaller than the Larmor gyro-radius. Medvedev (2001) proposed a composite model for GRB spectra by assuming the presence of a small scale magnetic field, which causes the jitter radiation component, and a larger scale field producing a standard synchrotron spectrum. The composite spectrum has a broad bump in correspondence of the jitter radiation characteristic energy, which depends only on the magnetic field properties and not on the electron energy and if the small scale field prevails, the spectrum has a limiting $\alpha=0$. This implies that only a minority of the hard spectra reported here could be consistent with such a model.

\subsection{Compton attenuation}

Compton attenuation (Brainerd et al. 1998) can produce a low energy (20-100 keV) hard component because of the propagation and scattering of the intrinsic spectrum by material (not participating to the bulk flow) located between the fireball and the observer. The original high energy spectral shape can be unaltered because of the decline of the Klein-Nishina cross section with energy, while at lower energies, where the scattering process is more efficient, the spectrum is hardened. The most appealing features of this model are that it could explain the clustering of the peak energy of bursts around some hundred $\mathrm{keV}$ and produce a flat slope at low energies. But in order for this to work, the Thomson optical depth of the scattering material must be quite large (of the order of $\tau_{\mathrm{T}} \sim 10$ ) and this would smooth out the observed burst light curve by smearing the variability on the smallest time scales. Furthermore, by decreasing the transmitted (spectrally unaltered) flux, this mechanism requires intrinsic powers greater than what we observe, exacerbating the energy budget problem.

\subsection{Quasi-thermal comptonization}

The above models are based on the assumption that the emitting particles are highly relativistic, as a result of "instantaneous" acceleration at a shock front. However, if the heating mechanism operates on timescales of the order of the dynamical time (or the light crossing time of a shell in the internal shock scenario), then the heating and cooling processes could balance, leading to typical energies of the electrons that are sub or only mildly relativistic (Ghisellini \& Celotti 1999). In this case the dominant radiation process is Comptonization of seed soft photons by a quasi-thermal particle distribution.

Different models assume different sources for the seed photons and different parameters (i.e. typical size for the emitting region, magnetic field, role of the photospheric radiation, and so on) within the internal shock scenario.

Liang et al. (1997), among the first to propose such process for the prompt emission, considered a relatively large emitting region embedded in a relatively weak magnetic field. 
This model was applied to GRB 990123 by Liang (1999), who found quite extreme best fit values for the required total power and number of emitting particles. Ghisellini \& Celotti (1999) proposed that Comptonization acts on the self-absorbed cyclosynchrotron radiation produced by the same quasi-thermal particles responsible for the multiple scattering process, and pointed out the role that electron-positron pairs can have in keeping the temperature (or the mean energy) of the particles within a narrow range. On the same line Mészáros \& Rees (2000) pointed out the importance of the residual photospheric emission as source of soft photons.

The basic features of the Comptonization model is that, in the quasi-saturated regime a Wien peak with its characteristic $F(E) \propto E^{3}$ shape can form at high energies (i.e. for $h v \sim k T$ ). In principle this can thus explain very hard spectra. One possible difficulty of this model is the slope at lower energies, whose saturated value should be $\alpha=-1$. A further possible problem for quasi-thermal Componization is that the temperature of the emitting particles (in the comoving frame) is expected to be of order of $\sim 50 \mathrm{keV}$, which would lead to an observed $E_{\text {peak }}$ of few MeV (Ghisellini \& Celotti 1999). This is a somewhat high value, also in the light of the results presented in this work, which show that even spectra time resolved on scales $\leq 1 \mathrm{~s}$ present a peak energy of $E_{\text {peak }} \leq 500 \mathrm{keV}$.

\subsection{The possible thermal character of the initial phase}

The first $1-5 \mathrm{~s}$ of the emission of the bursts presented in this work were found to be consistent with a black body spectrum with typical temperatures initially around $\sim 100 \mathrm{keV}$ and decreasing to $30-40 \mathrm{keV}$. After this initial phase a softer (non-thermal or multi-temperature) character of the spectrum becomes dominant. Clearly this behavior can be a powerful diagnostic. In particular it seems to favour two among the proposed models, namely photospheric and Compton drag emission, which both predict - as recalled in Sects. 5.5 and 5.6 - a thermal spectrum during the first phases. Here we briefly discuss which physical constraints can be quantitatively gathered from the data.

\subsubsection{Photospheric emission}

When the fireball is becoming optically thin, during the acceleration or coasting phase, its internal energy is emitted with a black body spectrum whose observed temperature is blueshifted by a factor $\Gamma$ with respect to the comoving one. The possible presence of a photospheric component in the spectrum of GRBs has been theoretically investigated by Mészáros \& Rees (2000). Recently, Daigne \& Mochkovitch (2002) have reconsidered this possibility, finding quite tight limits on the model of hot fireballs accelerated through internal radiation pressure, imposed by the absence of an initial emission phase (or precursor) with a black body shape.

In this scenario we have two options: we can relate the initial phase to a single fireball, in the process of becoming optically thin, or - following Daigne \& Mochkovitch (2002) - to an ensemble of $\mathcal{N}$ shells, each becoming optically thin at approximately the same distance. In both cases at transparency:

$\tau_{\mathrm{T}}=\frac{E_{\mathrm{f}} \sigma_{\mathrm{T}}}{2 \pi \theta^{2} R_{\mathrm{t}}^{2} m_{\mathrm{p}} c^{2} \Gamma \mathcal{N}} \sim 1$,

where $R_{\mathrm{t}}$ is the transparency radius of the fireball, collimated into two cones of semi-aperture angle $\theta, E_{\mathrm{f}}$ is the total fireball energy (a fraction $\epsilon_{\gamma}$ of which is radiated as photons), and $\sigma_{\mathrm{T}}$ is the Thomson cross section. Assuming that the surface of the fireball is emitting, at $R_{\mathrm{t}}$, as a black body with a comoving temperature $T^{\prime}=T_{\text {obs }} / \Gamma$, we have

$\left\langle L_{\mathrm{BB}}\right\rangle=\frac{\epsilon_{\gamma} E_{\mathrm{f}}}{t_{\mathrm{BB}}}=2 \pi \theta^{2} R_{\mathrm{t}}^{2} \sigma\left(\frac{T_{\mathrm{obs}}}{\Gamma}\right)^{4} \Gamma^{2}$,

where $\left\langle L_{\mathrm{BB}}\right\rangle$ is the average black body luminosity in the observer frame for the time $t_{\mathrm{BB}}$ and $\sigma$ is the Stefan-Boltzmann constant. Both Eqs. (2) and (3) show a $E_{\mathrm{f}} \propto \theta^{2} R_{\mathrm{t}}^{2}$ dependence, allowing to solve for the bulk Lorentz factor:

$$
\begin{aligned}
\Gamma & =\left(\frac{\sigma_{\mathrm{T}} \sigma}{m_{\mathrm{p}} c^{2}} \frac{T_{\mathrm{obs}}^{4} t_{\mathrm{BB}}}{\epsilon_{\gamma} \mathcal{N}}\right)^{1 / 3} \\
& \sim 3 \times 10^{3} T_{\mathrm{obs}, 9}^{4 / 3} t_{\mathrm{BB}}^{1 / 3} \epsilon_{\gamma}^{-1 / 3} \mathcal{N}^{-1 / 3}
\end{aligned}
$$

with $T_{\text {obs }}=10^{9} T_{\text {obs }, 9} \mathrm{~K}$ (corresponding to the observed black body peak of $\sim 100 \mathrm{keV})$. We stress that this estimate of $\Gamma$ is independent of the degree of collimation of the fireball.

In the case of a single shell $t_{\mathrm{BB}}$ is the time needed for the fireball to become transparent, implying

$R_{\mathrm{t}}=c t_{\mathrm{BB}} \Gamma^{2} \sim 3 \times 10^{17} T_{\mathrm{obs}, 9}^{8 / 3} t_{\mathrm{BB}}^{5 / 3} \epsilon_{\gamma}^{-2 / 3} \mathrm{~cm}$.

Therefore, if the emission comes from a single shell, the transparency radius is very large, implying unreasonably large values for the luminosity and energy, i.e. from Eq. (3), $E_{\mathrm{f}} \sim$ $3 \times 10^{58} \theta_{-1}^{2} T_{\text {obs } 9}^{20 / 3} t_{\mathrm{BB}}^{11 / 3} \epsilon_{\gamma}^{-5 / 3}$ erg, where $\theta$ is expressed in units of 0.1 radians.

If, instead, the observed emission comes from a series of shells the transparency radius can be much smaller, allowing a "reasonable" luminosity of the photosphere: for $E_{\mathrm{f}}=10^{51} E_{\mathrm{f}, 51}$ erg we have

$R_{\mathrm{t}} \sim 5 \times 10^{13} \frac{E_{\mathrm{f}, 51}^{1 / 2}}{\theta_{-1} \mathcal{N}^{1 / 3}}\left(\frac{\epsilon_{\gamma}}{t_{\mathrm{BB}} T_{\mathrm{obs}, 9}^{4}}\right)^{1 / 6} \mathrm{~cm}$.

We conclude that a series of shells (of total energy $E_{\mathrm{f}}$ ) each becoming transparent at $R_{\mathrm{t}}$ can account for the observed black body emission. The case of $\mathcal{N}$ shells for large $\mathcal{N}$ is clearly equivalent to a continuous or quasi-continuous flow.

As Daigne \& Mochkovitch (2002) pointed out, and in agreement with our findings, the photospheric radiation is likely to be visible only during the first phases of the burst light curve, as long as the optical depth of the material ahead of the shell which is releasing its thermal radiation is negligible and before internal shocks take over. 


\subsubsection{Compton drag}

If the circum-burst environment is characterized by quite a large photon density, as is the case of bursts following a supernova explosion, or for fireballs produced in the matterevacuated funnel of an hypernova, there can be a strong interaction between these seed photons and the fireball itself, as postulated in the so-called Compton drag model. The ambient photon energy is boosted by the factor $\Gamma^{2}$ at the expense of the fireball kinetic energy (Lazzati et al. 2000). If the funnel or the young supernova remnant are characterized by a single temperature $T_{\mathrm{SN}}$, and if the fireball does not decelerate, the emitted spectrum is a black body at a temperature $\sim 2 \Gamma^{2} T_{\mathrm{SN}}$. If instead the seed photons have a range of temperatures (as likely to be in the case of a funnel, hotter in the central parts), and/or the process is so efficient to decelerate the fireball, than the final spectrum will be a superposition of the locally produced black body spectra, as calculated by Ghisellini et al. (2000). The resulting spectrum can indeed resemble the very hard spectra found, at least for the initial part of these bursts. In fact, once the first shells have swept up the seed photons, the efficiency of Compton drag is greatly reduced, since the time to replenish the circum-burst environment with seed photons is typically longer than the duration of the burst itself. On the other hand the Compton drag model favours the formation of internal shocks, since after the first shells deceleration, the subsequent ejecta can more easily collide with them and produce shocks. Then another radiation source can become efficient after the first phase (lasting $\sim 1$ to a few seconds). This possibly also explains the hard to soft evolution: at first the spectrum, due to Compton drag, is very hard at low energies $\left(F(E) \propto E^{2}\right)$, whereas at later times the spectrum produced in internal shocks through other processes becomes softer $\left(F(E) \propto E^{1 / 3}\right.$ or $\left.\propto E^{0}\right)$.

Let us then assume that the time interval for which the black body lasts corresponds to the emission from a single shell and that the emission peaks when the shell becomes transparent (Eq. (2)), implying $R_{\mathrm{t}} \sim c t_{\mathrm{t}} \Gamma^{2}$.

The total energy $E_{\mathrm{CD}}$ due to the Compton drag process, produced in the observed time $t_{\mathrm{t}}$, is of the order

$$
\begin{aligned}
E_{\mathrm{CD}} & \sim 2 \pi \theta^{2} R_{\mathrm{t}}^{3}\left(2 \Gamma^{2}\right) a\left(\frac{T_{\mathrm{obs}}}{2 \Gamma^{2}}\right)^{4} \\
& \sim 3 \times 10^{51} \theta_{-1}^{2} t_{\mathrm{t}}^{3} T_{\mathrm{obs}, 9}^{4} \text { erg }
\end{aligned}
$$

where $a=7.56 \times 10^{-15} \mathrm{erg} \mathrm{cm}^{-3} \mathrm{~K}^{-4}$ is the radiation constant. In this form $E_{\mathrm{CD}}$ does not depend on $\Gamma$ (although of course $T_{\mathrm{SN}}$ does). We can then derive the transparency radius (from Eqs. (2) and (8)) and the bulk Lorentz factor:

$$
\begin{aligned}
R_{\mathrm{t}} & =\left(\frac{\sigma_{\mathrm{T}} c^{3 / 2} t_{\mathrm{t}}^{7 / 2} a T_{\mathrm{obs}}^{4}}{8 \epsilon_{\gamma} m_{\mathrm{p}}}\right)^{2 / 5} \\
& \sim 3 \times 10^{14} T_{\mathrm{obs}, 9}^{8 / 5} t_{\mathrm{t}}^{7 / 5} \epsilon_{\gamma}^{-2 / 5} \mathrm{~cm}
\end{aligned}
$$

$\Gamma=\left(\frac{R_{\mathrm{t}}}{c t_{\mathrm{t}}}\right)^{1 / 2} \sim 100 T_{\text {obs }, 9}^{4 / 5} t_{\mathrm{t}}^{1 / 5} \epsilon_{\gamma}^{-1 / 5}$

for a temperature of the seed photons corresponding to $T_{\mathrm{SN}} \sim$ $5 \times 10^{4} \mathrm{~K}$.

These simple (and rough) estimates indicate that the Compton drag model is viable.

\subsubsection{Time evolution}

Although the limited number of time resolved spectra and bursts do not allow to search for a general evolutionary behaviour, we can briefly comment on the spectral and dynamical evolution of the initial thermal phase of these 5 bursts. As reported in Fig. 10 in all the 5 bursts, the black body temperature decreases with time (bottom panel) and its evolution is intriguingly close to $T_{\mathrm{obs}} \propto t^{-1 / 4}$ for the first few seconds, and then drops. In the same time interval the luminosity $\left(L_{\mathrm{BB}}\right)$ remains constant or mildly increases (top panel of Fig. 10). We therefore observe, at least in the first phase, a decrease in the observed temperature without a corresponding decrease in the observed black body flux. We plan to examine more deeply these observational behaviour in a future work: here we just briefly comment on it in relation to the scenarios discussed above.

In the context of the photospheric model discussed in Sect. 5.7.1, the black body evolution could be explained by successive shells having an increasing baryon loading and smaller Lorentz factor, thus becoming transparent at increasing distances $R_{\mathrm{t}}$. This can cause the observed temperature to decrease (because the Lorentz factor is smaller) without a decrease in flux (because the radius is larger).

In the context of the Compton drag model, a decrease in the observed temperature can be due to the deceleration of the fireball and/or a decreasing temperature of the seed photons with distance. If we consider the luminosity due to the Compton drag process (Eq. (8)), we have $L_{\mathrm{CD}} \propto E_{\mathrm{CD}} / t \propto t^{2} T_{\mathrm{obs}}^{4}$, which predicts $L_{\mathrm{CD}} \propto t$ if the time behavior of the observed temperature is indeed $T_{\mathrm{obs}} \propto t^{-1 / 4}$. A weaker dependence of the observed flux on time can occur if the fireball is becoming transparent (i.e. only a fraction $\tau_{\mathrm{T}}$ of the seed photons can be scattered) with $\tau_{\mathrm{T}}$ decreasing with distance and/or if the fireball distance becomes larger than the typical dimension occupied by the seed photons.

\section{Conclusions}

We have presented the spectral evolution of GRB 911118 and of a new case of hard burst, GRB 980306, together with some other hard bursts already reported in the literature. Their low energy spectral component is harder than $N(E) \propto E^{0}$ for a considerable period of their main peak emission and even after this initial phase their spectrum remains harder than the synchrotron limit $E^{-2 / 3}$. We have applied different tests to verify the significance of the low energy hardness, concluding that these results are indeed robust.

These GRB prompt spectra represent a challenge for the proposed emission scenarios as shown through a comparison with the limiting spectral shapes predicted by such models. We pointed out the difficulties that synchrotron emission, even including the effects of self-absorption, small pitch angles particle distributions and jittering, has in explaining spectra harder than $N(E) \propto E^{0}$. Comptonization models also have difficulties, even if they are consistent with very hard spectra in a limited range of energies. Of course, such a pruning of the forest of emission scenarios refers only to the phases characterized by the harder emission, while it is possible and likely that different 
processes dominate at different stages of the prompt evolution and/or in different GRBs (see also below).

The main result of this study is the possible thermal character of the first emission phase of all the bursts we considered and the findings on the evolution of such a thermal phase. The conventional scenario, of a fireball accelerated by its own internal pressure, indeed predicts such an initial thermal character when the fireball becomes transparent. It was indeed its absence in previously considered bursts that led Daigne \& Mochkovitch (2002) to favour a rather cold fireball scenario, where at least part of the acceleration was due to magnetic forces. In our bursts, on the contrary, the luminosity in the thermal phase is a significant fraction of the total, therefore in agreement with the hot fireball scenario. However, the fireball could be cold initially, and be heated later (but before it becomes transparent). The heating agent could be magnetic reconnection (see e.g. Drenkhahn \& Spruit 2002) or internal shocks occurring at early phases. The latter case demands that the typical bulk Lorentz factors of the shells are small enough for the shells to collide before the transparency radius.

Alternatively, a cold fireball could work if the thermal spectrum we see is produced by Compton drag, in which circumburst radiation is boosted to high energies by the fireball bulk motion.

At present, we are not able to discriminate between the photospheric and the Compton drag scenario. However, a key difference between the two scenarios is the fact that the seeds photons for the Compton drag process can be "used" only by the first shells, because the time needed to refill the scattering zone with new seeds exceeds the duration of the burst. Therefore observing blackbody emission for a long time, or during the rising phase of two time resolved peaks would be difficult to explain in terms of the Compton drag process. We finally note that emission at times greater than a few seconds can well be due to other processes, possibly linked to internal shocks starting to dominate at later phases.

Acknowledgements. We thank the anonymous referee for a careful and thorough work and her/his constructive criticisms. This research has made use of data obtained through the High Energy Astrophysics Science Archive Research Center On line Service, provided by the NASA/Goddard Space Flight Center. G. Ghirlanda and AC acknowledge the Italian MIUR for financial support.

\section{References}

Amati, L., Frontera, F., Costa, E., et al. 1999, A\&AS, 138, 403 Band, D. L., Matteson, J. L., \& Ford, L. A. 1993, ApJ, 413, 281 Bevington, P. R., \& Robinson, D. K. 1992, Data reduction and error analysis for the physical science (2nd ed., McGraw-Hill)

Blinnikov, S. I. 1999, Astron. Rep., 43, 739

Böttcher, M., Dermer, C. D., Crider, A. W., \& Liang, E. P. 1999, A\&A, 343,111
Borgonovo, L., \& Ryde, F. 2001, ApJ, 548, 770

Brainerd, J. J. 1994, ApJ, 428, 21

Brainerd, J. J., Preece, R. D., Briggs, M. S., Pendleton, G. N., \& Paciesas, W. S. 1998, ApJ, 501, 325

Crider, A., Liang, E. P., \& Preece, R. D. 1997a [astro-ph/9711100]

Crider, A., Liang E. P., \& Smith, I. A. 1997b, ApJ, 479, L39

Crider, A., Liang, E. P., \& Preece R. D., et al. 1999, ApJ, 519, 206

Crider, A., \& Liang, E. P. 1999, A\&AS, 138, 405

Crider, A., \& Liang, E. P. 2000, ApJ, 127, 283

Daigne, F., \& Mochkovitch, R. 2002, MNRAS, 336, 1271

Dermer, C., \& Böttcher, M. 2000, ApJ, 534, L155

Drenkhahn, G., \& Spruit, H. C. 2002, A\&A, 391, 1141

Drenkhahn, G. 2002, A\&A, 387, 714

Ford, L. 1993, A Guide to Spectroscopic Oriented Analysis Routines, v. 1.21993

Ford, L. A., Band, D. L., \& Matteson, J. L. 1995, ApJ, 439, 307

Frontera, F., Amati, L., Costa, E., et al. 2000, ApJS, 127, 59

Ghirlanda, G., Celotti, A., \& Ghisellini, G. 2002, A\&A, 393, 409

Ghisellini, G., Lazzati, D., Celotti, A., \& Rees, M. J. 2000, MNRAS, 316, L45

Ghisellini, G., \& Celotti, A. 1999, ApJ, 511, L93

Ghisellini, G., \& Svensson, R. 1991, MNRAS, 252, 313

Goodman 1986, ApJ, 308, L47

Granot, J., Piran, T., \& Sari, R. 2000, ApJ, 534, L163

Katz, J. I. 1994, ApJ, 432, L107

Lazzati, D., Ghisellini, G., Celotti, A., \& Rees, M. J. 2000, ApJ, 529, L17

Lloyd, N. M., \& Petrosian, V. 2000, ApJ, 543, 722

Lloyd, N. M., \& Petrosian, V. 2002, ApJ, 565, 182

Liang, E., Kusunose, M., Smith, I. A., \& Crider, A. 1997, ApJ, 479, L35

Liang, E. 1999, A\&ASS, 138, 529

Medvedev, M. V. 2001 [astro-ph/0001314]

Mészáros, P., \& Rees, M. J. 2000, ApJ, 530, 292

Paczynski, B. 1986, ApJ, 308, L43

Panaitescu, A., \& Mészáros, P. 2000, ApJ, 544, L17

Papathanassiou, H. 1999, A\&AS, 138, 525

Preece, R. D., Briggs, M. S., Pendleton, G. N., et al. 1996, ApJ, 473, 310

Preece, R. D., Pendleton, G. N., Briggs, M. S., et al. 1998a, ApJ, 496, 849

Preece, R. D., Briggs, M. S., Mallozzi, R. S., et al. 1998b, ApJ, 506, L23

Preece, R. D., Briggs, M. S., Mallozzi, R. S., et al. 2000, ApJS, 126, 19

Preece, R. D. 2001, submitted

Preece, R. D., Briggs, M. S., Mallozzi, R. S., et al. 2002, ApJ, 518, 1248

Rees, M. J., \& Mészáros, P. 1994, ApJ, 430, L93

Ruffini, R., Bianco, C. L., Chardonnet, P., Fraschetti, F., \& Xue, S.-S. 2002, ApJ, 581, L19

Rybicki, G. B., \& Lightman, A. P. 1979 in Radiative Processes in Astrophysics (New York: Wiley)

Ryde, F., \& Svensson, R. 1999, ApJ, 512, 693

Ryde, F., \& Svensson, R. 2002, ApJ, 566, 210

Tavani, M. 1996, ApJ, 466, 768

Zhang, B., \& Mészáros, P. 2002, ApJ, 518, 1236 